This item was submitted to Loughborough's Research Repository by the author.

Items in Figshare are protected by copyright, with all rights reserved, unless otherwise indicated.

\title{
A comparison of digital and conventional sketching: Implications for conceptual design ideation
}

PLEASE CITE THE PUBLISHED VERSION

http://dx.doi.org/10.1504/JDR.2016.077028

PUBLISHER

(C) Inderscience

VERSION

AM (Accepted Manuscript)

\section{PUBLISHER STATEMENT}

This work is made available according to the conditions of the Creative Commons Attribution-NonCommercialNoDerivatives 4.0 International (CC BY-NC-ND 4.0) licence. Full details of this licence are available at: https://creativecommons.org/licenses/by-nc-nd/4.0/

\section{LICENCE}

CC BY-NC-ND 4.0

\section{REPOSITORY RECORD}

Self, James, Mark Evans, and Eunjin Kim. 2019. "A Comparison of Digital and Conventional Sketching: Implications for Conceptual Design Ideation”. figshare. https://hdl.handle.net/2134/20771. 


\title{
A Comparison of Digital and Conventional Sketching: Implications for conceptual design ideation
}

\author{
James Self* \\ School of Design and Human Engineering, \\ UNIST (Ulsan National Institute of Science \& Technology), \\ 50-UNIST gil, Ulsan \\ 689-798, Korea \\ Email: jaself@unist.ac.kr \\ *Corresponding author
}

\section{Mark Evans}

Loughborough Design School, Loughborough University,

Leicestershire,

LE11 3TU, UK

Email: M.A.Evans@lboro.ac.uk

\section{EunJin Kim}

Department of Industrial Design

KAIST (Korean Advanced Institute of Science \& Technology)

291 Daehak-ro, Daejeon

34141, Korea

Email: kejkim8@kaist.ac.kr

\begin{abstract}
The study compared the use of digital and conventional sketching as influence upon conceptual design work. Results show a significantly increased focus of attention upon the sketching tool in the digital condition compared to conventional sketching with pen and paper. Both a statistical analysis and fine-grained qualitative comparison illustrate how increased frequencies of sketching tool focused activities resulted in both significantly more time spent in problem orientated work and the fragmentation of attempts to transition from problem definition to generative solution ideation. In contrast, reduced tool-focused activity in the conventional sketching condition appeared to provide opportunities for significantly increased solution ideation through design-driven moves to explore reflect upon and develop solution propositions. The implications of a tool orientated focus of attention are discussed in terms of its impact upon reflective solution ideation during conceptual design.
\end{abstract}

Keywords: design activity, design sketching, reflective-practice, conceptual design ideation, design representation

Biographical notes: Dr. Self is Assistant Professor and Director of the Design Practice Research Lab (dpr Lab) at the School of Design and Human Engineering, UNIST. Dr. Self holds a doctorate in industrial design practice. Previous to this he worked for several years within the design industry. Adopting research on/for and through design approaches current and past work explores design skills, reasoning and practices, design innovation and design form and aesthetic. $\mathrm{He}$ is peer reviewer for a variety of international conference and journal publications and associate editor of Archives of Design Research and the Principles \& Practices collection.

Dr Mark Evans is a Reader in Industrial Design and leader of the Design Practice Research Group. A PhD supervisor and examiner for 25 candidates with over 100 publications and 
outputs as app, web site, video, museum/gallery exhibition, cards, PDF download, product; research activity focuses on supporting creative design practice through the development of tools/resources and exploring the impact of emerging digital technologies. Outcomes from recent research projects include the iD Cards design tool app for the Industrial Designers Society of America and 3D printed concrete exhibition for the UK's National Centre for Craft and Design.

Ms. EunJin Kim is a doctoral researcher based at the Color Lab, ID KAIST. After completing a Masters in design that both defined and examined the implications of cognitive design styles for conceptual design ideation, Ms. Kim's doctoral research aims to explore relationships between colour and affective response in the design context. She has already achieved publication success through paper presentations at international design conferences and peer reviewed design journals.

\section{Introduction}

To date, there has been a significant body of work comparing the influence of computer aided concept design tools with their conventional, non-digital counterparts. These studies have, for example, extended understanding of the media of representation as influence upon conceptual design ideation. However, a review of the literature indicates disagreement on the influence of digital design tools in their effectiveness as support for conceptual design activity. For example, Tang et al's (2011) protocol study, based upon a rationalist approach to the investigation of design practice (Gero and Mc Neill 1998), deployed the function-behaviour-structure framework proposed by Gero and McNeill (1998) in a statistical analysis of conventional compared to digital sketching tools. Tang et al's (op cit) study concludes that digital sketching media had no significant influence on the nature of design activity during conceptual ideation. In contrast, Stones et al. (2007, 2010) explored the sketching work of students when using digital and conventional media to assess implications for the reinterpretation of design ideas during conceptual ideation. That is, the extent to which the sketching tool provided opportunities to reflect upon solution attempts; revise and develop them based on an emerging understanding of the design problem. Their studies concluded that conventional sketching is more effective in supporting both reinterpretation and the ubiquitous generation of design ideas.

In contrast, Jonson (2005) conducted a case study analysis of five undergraduate design students and five design practitioners from various disciplines to examine the role and use of sketching during conceptual ideation. Adopting an in-the-wild-approach, the design participants' use of conceptual design tools was recorded through self-report diary entries that sort to capture both the frequency with which subjects used conceptual tools together with the reasons for their use. Results indicated a range of primary conceptual ideation tools most often used by the participants, with the exception of sketching. Further, according to the approach taken, the spoken and written word was found to be most frequently used during ideation compared to sketching and CAD tools, reported to be least frequently employed. Although results indicated a contradiction with the prevailing literature on the role and importance of sketching, Jonson (ibid) qualifies his findings by suggesting how participants 
considered their limited use of the tool a result of their particular design environment, rather than any personal rejection of the tool itself.

Building upon these existing studies, the current investigation examines how focus of attention between sketching tool and actual design work implicates reflection-in-action during conceptual design ideation.

\section{Reflection-in-Action}

In response to a rational approach to the examination of design practice (Simon 1996, Gero and Mc Neill 1998), Schon (op cit) describes the limitations of the rational problem solving paradigm in its ability to fully explain the nature of professional activities as they are experienced. The epistemology of reflection-in-action is proposed as a means to understand the kinds of experiential knowledge deployed in an artistry of professional activity, including design practice. This approach has since been taken up by the design research community in studies of design activity in contrast to the rational approach or a science of design (Dorst and Dijkhuis 1995, Dorst 1997). In essence, reflective practice describes a constructed, situated and embodied account of designerly activity (Dorst and Dijkhuis, 1995). Design is seen as a reflective conversation with the situation (Schon and Wiggins 1992, Lawson 2004) as unique problems in unique situations are framed by the designer or design team (Stolterman 2008, Nelson and Stolterman 2012). Moves are then made to explore, test and reframe the design problem through solution-focused reflection-in-action. Through this reflective act and in response to often ill-defined design problems (Rittel and Webber 1973), new insights are developed for the improvement of the current situation. Dorst and Dijkhuis (1995), in a comparison of the rational problem solving paradigm (Simon 1996) with the Schönian theory of reflective-practice (Schon 1983, Schon and Wiggins 1992), summarise the two approaches in terms of the designer, the nature of the design problem, the character of the design process and the kinds of knowledge it involves.

Similarly to existing design practice research (Schon and Wiggins 1992, Valkenburg and Dorst 1998, Goldschmidt and Smolkov 2006, Bar-Eli 2013), the current study's investigation of digital and conventional sketching employs the discursive epistemology of ways-of-knowing in professional practice first proposed by Schon $(1983,1987)$ and Schon and Wiggen $(1983,1987,1992)$ and further developed as a means to understand design practice by Dorst and Dijkhuis (1995) and Valkenburg and Dorst (1998). As such, the current study explores how the use of digital sketching may influence focus of attention between sketching as tool of design representation and actual design-driven work. We then examine the implications of a focus of attention upon sketching tool for engagement in problem definition (naming/framing) and solution ideation (moving/reflecting). Finally we discuss the influence of tool-focused work for reflection-in-action. The fact that our results appear to contradict those of others (Jonson 2005, Tang, Lee et al. 2011) provides further grounds for debate around the influence of digital sketching tools, their potential influence upon attention focus and implications for reflective practice. 


\section{Tools of Design Representation}

The current study examines how state-of-the-art digital sketching tools, as tool for design representation, influenced focus of attention between the tool (Self, Evans et al. 2014) and actual design activity. During conceptual design ideation the designer is required to propose and develop transformative solution ideas in response to often ill-defined or uncertain design problems (Rittel and Webber 1973, Dorst 2006). To cope with the uncertainty of design problems, the designer will ubiquitously employ design representation as means to both help constrain or frame the initial problem and assess the potential of alternatives as offering opportunities for improvement.

Of course the representation of design solutions may take many forms, from spoken communication to written documentation, sketches and illustrations or some combination of all these approaches concurrently or synchronically. However, our current discussion of design representation focuses upon the iconic (Chandler 2002) or figural (Goldschmidt 1997) expression of solution ideas so often seen as synonymous with design activity. That is, representations, such as drawings and illustrations but also models and prototypes, that attempt to approximate the attributes of potential solution ideas on one or more dimensions of interest (Ulrich and Eppinger 2012). These design representations may take many forms and be achieved through the applications of numerous tools, methods, media and approaches (Pei, Evans et al. 2011). The current study examines sketching as one such ubiquitous type of design representation and the digital and conventional tools used in the construction of figurative sketch representations.

As such the current study uses the term tool of design representation as construct to indicate the media (tool) through which design representation is achieved (digital or conventional sketching). We examine how the tool may influence the designer's representation of design intent and implication for design ideation. In particular, we investigate how the tool of design representation may influence focus of attention between the functional use of the tool itself (tool-focus) and tool use as means to foster reflection-in-action in pursuit of appropriate solution ideas (design-focus). In this way we are particularly interested in the interaction between the participants and the design tool, and resulting implications for design representation as driver for conceptual design ideation.

Thus, we outline the scope of a definition of design representation to an act of representation that attempts to approximate the physicality, functionality or use of a solution candidate for the purpose of assessing the potential of conceptual design ideas. And a definition of tool of design representation as the media, method(s) and tools used to express design intent for the purpose of assessing the potential of possible solutions.

Adopting the reflection-in-action paradigm (Schon 1983, Schon and Wiggins 1992) we examine the influence of the use of digital and conventional tools upon design representation. To achieve this we analyse time spent in tool-focused work compared to design-driven activity in conventional and digital sketching conditions, with tool-focused work describing instances where participants are focused upon the tool, its use and functionality. In contrast, design-driven activity is defined by the application of the tool in the representation of ideas for the goal of proposing, developing and 
evaluating the potential of solution candidates as part of what Schon (op cit) describes as the situated activity of reflection-in-action.

\subsection{Design Sketching}

From the simple line-work of the idea sketch to high fidelity prototypes, the representation, development and communication of design ideas through sketching appears to be an essential tool supporting conceptual design ideation. As such, studying the role and use of sketching as tool of design representation, provides opportunities to develop understanding of both the influence of the expert use of sketching as a tool for conceptual design and sketching's relationship to the kinds of abductive (Dorst 2011) or appositional reasoning (CROSS 1990) required to productively navigate between design problem definition, solution proposition and development.

For example, in an early study, Tovey et al. (2000) explored the characteristics of CAD (Computer Aided Design) as influence on automotive conceptual design as compared to traditional drawing and illustration techniques. In order to understand the influence of various design representations upon communication during design development, Pei et al. (2008) developed a taxonomy of design representations (sketches, drawings, models and prototypes). Their classification system indicates how the characteristics of the various design representations influenced how design intentions are communicated and developed. An extension to Pei et al. (ibid) taxonomy has been proposed by Kim et al. (2013) who expand on the classification of conceptual design representation to account for how the designer's particular goal in the construction of a representation influences both tool choice and the characteristics of the design representation itself.

Goel (1995) identifies insights into the relationship between sketching as tool for representation and reasoning during conceptual design. Sketching’s ambiguous nature, semantic density and ability to provide opportunities for transformations between and among design ideas are offered as distinctive characteristics of sketching as design tool and reasons for its effectiveness in supporting conceptual design activity. In an extension to Goel's (ibid) work and through the development of a notation system related to design transformations, Do et al. (2000) interprets the designer's reasoning as part of an investigation focused on design drawings aimed at understanding the relationship between representation through drawing and its association to conceptual design practice. Like Goel (op cit), Do et al (ibid) indicate how representation through drawing provides the designer with opportunities to reflectively explore various conceptual design ideas, progressing those that are seen as most appropriate given an emerging understanding of the design problem.

A further example of the influence of sketching is presented by McGown et al (1998) who use sketch representation as research object to provide evidence of how conceptual design activity is enhanced by digital design tools. In contrast, Bilda et al’s (2006) protocol analysis of design ideation without the ability to represent design intent through sketching (literally blind-folding designer participants and asking them to address a typically ill-defined design problem) indicated that there was no significant difference between sketching and not sketching based upon an analysis of the 
appropriateness of conceptual design outcomes and the kinds of reasoning engaged in the sketching and non-sketching conditions. As such, the Bilda et al (ibid) study questions the importance of sketching as tool for conceptual design ideation in terms of its ability to provide improved opportunities to propose and explore design ideas. In contrast however, Bouchard et al's (2006) study of sketching during conceptual automotive design indicated the importance of the sketch as tool for visualisation, reflection and concept development. The sketch was observed to support a flow of information between external and internal mental representations. Similarly, Goldschmidt et al, in an influential series of early papers (Goldschmidt 1994, Goldschmidt 1997, Goldschmidt and Weil 1998), discuss the importance of sketching as means to support visual thinking during conceptual ideation. Sketching is defined as a clue finding activity through the representation of design ideas and the designer's germane of one's own visual thinking, on the fly and in situ. As such, sketching as tool for the expression and development of intent is described as critical in facilitating the kinds of visual thinking required during conceptual design ideation.

Although these existing studies have contributed to an understanding of the act of sketching, no work was identified that explored sketching media in terms of its influence of attention-focus and implication for reflective-practice. As reflective-practice is described as providing opportunities to engage in the assessment of potential design ideas through the media of expression (i.e. sketching), understanding how different sketching media implicate attention focus can provide evidence to suggest how the media of representation may influence the reflective conversation with the situation. If reflective-practice is important to design activity, disruption between designer and reflection on design (i.e. tool focused work), will then have potential implications for the progress of solution ideas. It is thus important to understand how different tools of design representation may implicate attention focus between tool and reflective design-focused work.

\subsection{Digital and Conventional Sketching}

Although no existing study was identified to focus upon focus of attention between tool and designdriven activity; implications for reflection-in-action and conceptual ideation, there has been some progress in developing an understanding of the use of digital sketching as tool for conceptual design. For example, Won's (2001) study to address a deficiency in research related to digital sketching tools concludes that cognitive behaviour, when using conventional media, is similar to behaviour when using digital sketching tools. Employing a coding scheme that draws on Goldschmidt's (1991) concepts of seeing-as and seeing-that, with the former describing what the designer sees in the embodiment of design intent and the later the ways in which the representation is interpreted, Won's (op cit) study identifies an increased frequency of cognitive shifts from thinking about important considerations to pay attention to (seeing-as) to a transition to consider their potential (seeing-that). However, unlike the current investigation of state-of-the-art digital sketching tools, these earlier studies were limited by their use of conventional command-based design software (graphics software, 3D CAD) together with a traditional user interface (mouse, pointer). 
Similarly to Won's investigation, Bilda and Demirkan's (2003) study employed protocols of six interior designers to examine frequencies of perceptual actions. These were shown to be higher during conventional sketching compared to digital work. Results suggested increased actions appeared to provide opportunities for greater exploration through more frequent attention shifts. In terms of the reflection-in-action epistemology of professional practice, Bilda and Demirkan's (ibid) findings appear to relate to an ability to transition to solution orientated design moves based upon a developing understanding of the design problem through both naming important considerations and framing approaches to solution development. That is, their study indicates how conventional sketching provided increased opportunities, through design-driven attention shifts, for the generative exploration of solution ideas.

In contrast, Jonson's (2005) study of practicing designers indicated the use of verbal communication and CAD as prominent ideation tools during conceptual design and development. Jonson's study of designers in practice concludes that a hybrid approach, including both digital and conventional tools, was in reality often employed at various times, with decisions being dependent upon design objectives. Likewise, Bilda et al (2006) questions the premise of sketching through an analysis of think-aloud protocols based upon Suwa et al's (1998) cognitive action coding scheme. The scheme measures design ideation as a series of cognitive actions, each with different objectives and interests that range across and between content related to problem definition and solution ideation. Findings indicated no difference between sketching and not sketching, although the study did conclude by reiterating the importance of sketching in terms of its ability to alleviate the cognitive load required during conceptual design.

Similar to Bilda et al's (op cit) method of analysis, Tang et al (2011) investigated conventional and digital sketching environments to evaluate their influence on design ideation, deploying the function-behavior-structure (FBS) approach to the analysis of think-aloud protocols. Based on the FBS method of analysis, Tang et al (op cit) concluded that, the digital sketching environment is similar to free-hand sketching in all significant aspects of the design process (Tang et al., p22). Likewise, the study also indicated that digital sketching had no influence on how design ideation progressed in terms of time spent in explorative ideation compared to problem definition. However, contradicting Tang et al's (ibid) findings, two further studies by Stones and Cassidy $(2007,2010)$ investigated levels of reinterpretation between digital and conventional sketching, where reinterpretation is described as, 'complex, bi-directional cognitive process' (Stones and Cassidy, p440). Both studies found that conventional sketching lead to increased reinterpretation, providing greater opportunities for the creation of new solution ideas. That is to say, conventional sketching as tool for design representation appeared to provide greater opportunities to reflect upon and interpret design intentions which allowed for a wider exploration of possible solution ideas.

\section{Aims}


We examine the implications of focus of attention for reflection-in-action in terms of the naming and framing of important considerations during problem definition and effect upon moving and reflecting activity during solution ideation. With these aims in mind, the current study addresses the following research question:

- What implications does focus of attention have for reflection-in-action during conceptual ideation?

\section{Methods}

We employ protocol analysis as means to investigate the influence of sketching condition upon toolfocused activity and then examine implications for reflection-in-action. Although numerous methods have been used to explore conceptual design activity (i.e. interview, observation, ethnography, diary studies), protocol analysis remains the most often employed approach to the examination of design practice (Chai and Xiao 2012). This is because it provides opportunities to gather data related to an individual's situated activity of design rather than reflecting upon design work after the event, recording events as they happen or attempting to interpret meaning from observation. As reflectivepractice is concerned with the experience of design as part of a situated activity, protocol analysis is particularly suitable for our examination of how sketching condition may influence focus of attention and implications for reflection-in-action.

The types of think-aloud experiment and variations in their format differ within the literature, from concurrent think-aloud to retrospective studies (Perry and Krippendorff 2013). The length of time provided for a think-aloud session also varies from several hours to as little as 15 minutes (Jiang 2009, Chai and Xiao 2012). As Perry and Krippendorff's (op cit) suggest, concurrent think-aloud may have implications for the subject's ability to perform the design task. As such, a retrospective approach was adopted. Participants were required to watch their own design activity immediately after the design session and concurrently tell the researcher what they were thinking as they engaged the design task.

\subsection{Participants}

A sample of ten $(n=10)$ final year undergraduate industrial design students was taken from an established design school in the Republic of Korea. Subjects were chosen from a student cohort according to their ability to fulfill the following three criteria for selection:

1. Successful completion of a Design Fundamentals and Sketching Skills module

2. Enrollment as a full-time student in the final year of a four year degree programme

3. Currently studying within the Industrial Design Department

Criteria 1 and 2 were used to ensure an appropriate degree of sketching and design capability within the sample. This was important due to the focus of the study on the design tools as opposed to a focus on an ability to sketch. As such, the study required participants with similar sketching and 
design abilities to limit the influence of experience/sketching skill and focus on implications of the two sketching conditions as object of study. According to the Dreyfus and Dreyfus (1980) model of skills acquisition, while not constituting an expert level of design expertise, the participants' design knowledge and skills could be described as competent (Table 1).

Table 1 Attributes of sample of competent designers (As instructed, please find all tables and figures appended to end of manuscript)

While not yet expert, having taken design studio classes, design project courses, sketching and illustration skills classes over the three years of their degree to the point of their participation, participants possessed a working knowledge of design practice. Moreover, all participants had industry work experience as interns on design projects with industry either within the institution or as industry interns as part of their undergraduate education. As such, they were able to cope with the complexities of design problems and approach design tasks in terms of longer-term goals.

\subsection{Study task environment}

The subjects' response to a given conceptual design problem (see below) were recorded through three video recorders (Figure 1).

Figure 1 Task environment

Camera 01 recorded an overview of the environment; camera 02, overlooking the participants, recorded activity performed within the subjects' working environment (sketching, drawing, writing, and reading); Camera 03 was positioned to record the subjects' movements and body posture as the design task was completed. During conventional sketching protocol sessions, participants were provided with plain drawing paper, pens, pencils, marker pens and drawing templates. During the digital sessions, a Wacom Cintiq graphics tablet with pre-loaded Alias Sketchbook Pro Software was provided, giving participants digital sketching tools that had been developed to closely approximate the affordances of conventional sketching.

\subsection{Design Task}

In order to examine the influence of sketching conditions (conventional and digital) participants were presented with a design problem of similar complexity for each of the two sketching conditions. The first required the conceptual development of a sports watch for young people. The second asked subjects to develop a concept for a luxury watch for the same target user. Taking an experimental approach to the phenomena of design practice has the potential to de-contextualise what is a highly collaborative and context-driven process (Stolterman 2008). On the other hand, opting for an experimental methodology provides control of the environment, allowing a focus upon the potential influence of sketching condition upon focus of attention between design work and tool, thereby 
reducing much of the noise associated with 'in-the-wild' design research (Michel 2007). Moreover, given our analyses of the use of tool of design representation at a fine-level of granularity, the study required the inclusion of recording equipment which made the experimental design impractical for inthe-wild studies. As such, we follow methodological approaches to research in design practice as seen in such seminal works as Cross et al's original protocol workshops (1996) and Goel's (1995) sketching study.

\subsection{Task Procedure}

Participants were given instructions related to the aims of the research, their rights and obligations, task procedure, methods of recording and time given to complete the task. The researcher then withdrew from the environment, leaving subjects 25 minutes to respond to the design problem. Immediately after the task, the participants' recorded sketching activity was played back to them during retrospective think-aloud sessions. (Someren, Barnard et al. 1994). That is, directly after each design session, the participants' recorded design work was played back to them. During this time participants were asked to describe what they were thinking (think aloud) while watching the recording of themselves engaging the design task. This retrospective approach to think aloud sessions was adopted to avoid the problem of having to think aloud while engaging the design task when adopting a concurrent think aloud approach, thereby distracting participants from the design task. Each participant undertook the two design tasks alternately. To limit order effect, half the sample engaged the conventional condition first, while the other half employed digital sketching. Before each of the digital sessions, participants were provided with two hours of training to re-familiarise themselves with the digital tools (all students had full access to the digital tools within a digital design studio). The 20 task sessions involving the ten participants were completed at the researchers' institution over a period of 12 weeks.

\subsection{Analysis}

Sixteen of the 20 transcribed protocols were first encoded using Qualitative Content Analysis (QCA) software. After completion of two of the digital sketching sessions, a fault was discovered with the software used to capture sketching activity in the digital condition. As such two of the original ten participants were removed from the study. The transcribed think-aloud sessions were first segmented using thematic criterion. Two coders separately looked for phrases, sentences and discourse that appear to hang together as a single idea, action or thought. Following segmentation, coders assigned units of coding to the dimensions of a coding frame adapted from Valkenburg and Dorst's (1998) encoding classification system. The Valkenburg and Dorst (ibid, Table 2) provide four theoretical constructs through which units of coding were classified. The four concepts (naming, framing, moving and reflecting) originate from Schon's $(1983,1987)$ reflective practice epistemology, with various studies having since extended their meaning (for example (Dorst and Dijkhuis 1995, Gero and 
Kannengiesser 2008, Tang, Lee et al. 2011, Bar-Eli 2013). In alignment with the research question (the implications of attention focus for reflection-in-action during conceptual ideation), four reflectionin-action encoding categories were used in the encoding of the think-aloud segments. A summative description of each concept is provided in Table 2.

Table 2 Four conceptual coding categories

In addition to the four activity categories, a further encoding category: TDR-focused (Tool of Design Representation Focused) was added (Table 3). A focus upon TDR is described by Self et al. (2014) as a shift of attention from the activity of design to the tool of design representation itself (toolfocus). Similar to Stolterman et al's (2008) concept of tool-first/design-first approaches and Menezes and Lawson's (2006) notion of a designer's ability to, 'have a strong interaction with their own drawings' (Menezes and Lawson 2006, p583), a TDR-focus is identified as an explicit attention-shift from design work to the tool of design representation; its use, limitations, strengths and manipulation in the construction of design representations. The concept of being TDR-focused was added as a means to compare tool-focused and design-focused activity in the conventional and digital sketching conditions. Unlike the four reflection-in-action encoding categories (Table 2), encoding of segmented protocol data as TDR-focused was not exclusive (i.e. TDR-focused data was also encoded as naming or framing or moving or reflecting). This approach provided a means to examine differences in toolfocused activity between sketching conditions in terms of the four reflection-in-action activities of naming, framing, moving and reflecting. The additional TDR encoding category is identified in Table 3.

Table 3 TDR (Tool of Design Representation) coding category

In order to limit the inherent subjectivity required in any QCA, a segmented transcription was encoded by two coders separately. Encoding was then compared for consistency. Disagreements were examined and decision rules agreed upon and applied as required. That is, the two coder came together to present and discuss approaches to encoding of protocols in a qualitative way (Schreier 2012). For example, disagreement between coders on the encoding of the segmented protocols was then discussed to identify the source of disagreement in terms of an interpretation of the encoding category. Rules were then applied and/or the encoding frame revised. In this way, the reliability of the frame (its ability to classify the segmented protocol transcriptions) and validity (its ability to describe the participants design activity) were tested.

\section{Results}

In the following sections, the results of both a quantitative statistical analysis and qualitative comparative analysis are presented. Frequency counts of and transitions between the encoded reflection-in-action activities of naming, framing, moving and reflecting, together with TDR (tool- 
focused) and Non-TDR focused (design-focused) activities, are analysed to examine the effect of sketching condition on focus of attention and implications for reflection-in-action. The qualitative analysis of encoded protocols is then presented, supported by flow-chart illustration and images of design activity during digital and conventional sketching conditions to further examine the implications of tool-focused work upon conceptual design ideation.

\subsection{Quantitative comparison}

After encoding, the protocol frequencies of naming, framing, moving and reflecting activities were calculated together with the frequencies with which these activities were encoded as TDR (tool) or non-TDR (design) focused. Figure 4 presents encoding frequencies $(f)$ for all participants (A-J). Mean $(M)$ and standard deviation $(S D)$ provide an indication of dispersion of each category across the participants.

Figure 2 Frequencies of absolute encoding (f) for all participants (n) across coding frame dimensions

\subsection{Comparing TDR and non-TDR focused activities}

A Wilcoxon signed-rank test was run to determine whether there was a significant difference between conventional and digital sketching in terms of TDR (tool-focused) and non-TDR (design-focused) activities. As independent variable, type of sketching condition was employed (digital and conventional), with frequencies of TDR-focused and non-TDR-focused activities used as dependent variables. The result indicated the frequencies of TDR-focused activities were significantly higher in the digital sketching condition ( $M d n=51.50)$ compared to conventional sketching ( $M d n=19.50)$; $Z=2.52, p<.05$, and that non-TDR-focused activities were significantly increased in the conventional condition ( $M d n=72.00)$ compared to the digital ( $M d n=57.00) ; Z=-2.37, p<.05$ (Figure 3).

Figure 3 Frequencies of TDR and non-TDR focused activities between conventional and digital sketching.

These results indicated that, during the digital sketching condition, participants spent a significantly increased amount of time in tool-focused activities when compared with conventional sketching. The following sections indicate the implications of increased tool-focused work for distribution of and transitions between the reflection-in-action activities of naming, moving, reflecting and framing during the digital and conventional conditions.

\subsection{Comparing Distributions across Activities}

A Chi-square test was run with the distribution of eight activities in order to investigate the effect of sketching condition. Firstly, the distributions of the eight activities were compared (TDR and NonTDR-focused naming, framing, moving and reflecting). The type of sketching tool and the type of design activity were defined as independent variables, with frequencies of each activity defined as 
dependent variables. The results indicated that there was a significant difference between conventional and digital sketching conditions in terms of the eight design activities, $\left(\mathrm{X}^{2}=123.52 \mathrm{df}=7, p<.05\right)$ and in terms of the distribution of the design activities during TDR (tool) and non-TDR (design) focused naming, framing, moving, and reflecting.

These differences were further examined using Wilcoxon signed-rank tests. For each of the eight activities, frequencies within the conventional and digital sketching conditions were compared (Table 4). The results indicated significant differences in four activities between the two sketching conditions: non-TDR-focused naming, TDR-focused moving, TDR-focused reflecting and non-TDR focused reflecting.

Table 4 The results of Wilcoxon signed-rank tests for the frequencies of eight design activities

The analysis showed a significant difference in the frequencies of non-TDR focused naming activity between conventional sketching $(\mathrm{Mdn}=23.00)$ and digital sketching $(\mathrm{Mdn}=13.00) ; Z=-1.96, p<.05$. This result indicated that the frequencies of non-TDR naming activities decreases in the digital condition compared to conventional sketching, implying increased TDR-focused work in the digital condition resulted in significantly less non-TDR focused naming activity.

The use of the digital sketching tool also increased TDR-focused reflecting activity ( $M d n=13.00)$ compared to conventional sketching ( $\mathrm{Mdn}=7.50) ; Z=2.52, p<.05$, with the frequency of non-TDR focused reflecting activity decreasing during digital ( $M d n=14.50)$ compared to conventional sketching (Mdn=16.50); $Z=-2.21, p<.05$. Figure 4 presents the box plots of the four activities: Non-TDR focused naming, TDR-focused moving, TDR-focused reflecting and non-TDR focused reflecting; clearly illustrating differences between the two sketching conditions.

Figure 4 Box plots of frequencies of four activities showing significant difference between conventional and digital sketching

In summary, a significantly increased tool-focus reduced the participants' design orientated naming of considerations within the problem space; increased activity associated with moving to resolve tool related issues (tool-focused moving); increased reflections on the suitability of the tool in its ability to accomplish a given task (tool-focused reflecting), and significantly reduced design focused reflections upon the suitability of conceptual ideas.

\subsection{Comparing Transitions between Activities}

In an analysis of transitions, TDR-focused and non-TDR focused activities were aggregated together in order to minimize the number of independent variables. This resulted in a total of sixteen types of transitions, as listed in Table 5. Differences between distributions of all 16 types of transitions were compared using a Chi-square test. The results indicated that there was a significant effect of sketching condition on the distributions of transitions among naming, framing, moving and reflecting, $\left(\mathrm{X}^{2}=\right.$ 36.15, $\mathrm{df}=15, p<.05)$. 
Table 5 Numbers of transitions in 8 participants for conventional and digital sketching

Differences between conventional and digital sketching were further examined for each type of transition using Wilcoxon signed-rank tests. Among 16 transition types, results indicated that differences in sketching condition had a significant effect on the number of transitions between moving to moving, moving to reflecting, and reflecting to moving. Figure 5 presents box plots of these three significant results.

Figure 5 Box plots of transitions showing significant differences for conventional and digital sketching.

The use of digital sketching significantly increases the number of transitions between moving and moving ( $M d n=19.00)$ compared to conventional sketching ( $M d n=12.50) ; Z=2.37, p<.05$. There were increased transitions from moving to reflecting in the digital sketching condition ( $\mathrm{Mdn}=17.50)$ compared to conventional sketching ( $\mathrm{Mdn}=13.50) ; \mathrm{Z}=2.18, p<.05$ ). Reflecting to moving also increased significantly when participants used digital sketching ( $\mathrm{Mdn}=15.50)$ compared to conventional sketching ( $\mathrm{Mdn}=9.00) ; \mathrm{Z}=2.38, p<.05)$.

In summary, results indicated how the use of digital sketching increased transitions between moving activity together with transitions between moving and reflecting, and reflecting and moving. In our approach to the analysis of transitions, it was unclear as to how a significantly increased toolfocus implicated statistically significant differences in transitions between the two sketching conditions. Table 6 summarises results from the statistical analysis of encoded protocol data.

Table 6 Summary of Results

\subsection{Qualitative Comparison of Sketching Conditions}

The following section further examines the influence of increased tool-focused work in the digital sketching condition upon attention focus and reflective-in-action during conceptual ideation. To achieve this, encoded protocol data of the participants' think aloud responses when engaged in a conceptual design task was subjected to a comparative, qualitative analysis. In doing we examine the implications of statistically significant differences in tool-focused vs. design-focused work, plus frequencies of and transitions between activities during conceptual design ideation.

Figure 8 graphically illustrates participant C's naming $(\mathrm{N})$, framing $(\mathrm{F})$, moving $(\mathrm{M})$ and reflecting (R) activity in both conventional and digital sketching conditions. Activities also encoded as TDR-focused (tool-focused) are shown in solid black and annotated with short descriptors.

Figure 6 Flow-diagram illustrating design activity of participant $\mathrm{C}$ with tool-focused activity in black

Tool-focused work

Participant C commences both conventional and digital protocols by naming things to pay attention to within the problem space, "I have to consider all these things" (conventional). However, within the digital condition's first five minutes (Figure 6, 0:00-5:00), C's sequence of naming and 
moving is interrupted by six tool-focus shifts i.e. naming and moving to identify tool functionality (Figure 6, black bars i.e. little training with tablet/software).

Indicating the implications of these six tool-focused events, in the third minute of her protocol, $\mathrm{C}$ defines the frame of analog display as possible partial solution candidate, "So I think analog provides a more luxurious feeling to us". However, rather than moving to test the frame's suitability, given an emerging understanding of the design problem, the protocol's fourth minute see's further tool-focus shifts (Figure 7, mistake with tool, inability to use tool, search for correct tool). Figure 7 illustrates C's working environment up to the digital protocol's fifth minute.

Figure 7 Screen-capture of working environment, 00:00-05:00 of digital condition

C's tool-focused shifts at the start of her digital protocol appear to slow the progress of transition from problem definition to solution ideation. Evidence of this was also observed in C's working environment at the end of the digital protocol's fifth minute (Figure 7). The word list (a hybrid of words and statements from the design brief and C's own initial thoughts) indicated she was yet to transition from the naming of potential issues within the problem space to the generation and exploration of possible solution candidates. By the end of the protocol's fifth minute, it appears her tool-focused activity has inhibited her ability to move from problem-focused naming to more generative, solution focused moving activity.

In contrast, at the start of the conventional condition's third minute, $\mathrm{C}$ continues her designfocused sketching, whereupon her sketch work acts as stimuli for strap design, "It will be a strap, kind of a strap. What could be the best for this design, proper for this design?" As evidence of the influence of increased framing transitions identified in the statistical analysis, it is at this point that a clipping watch-clasp emerges as a partial-solution frame (Figure 8, framing activity). C then immediately spends the next minute engaged in design-focused moving through sketching as means to test the frame's suitability against her emerging understanding of the design problem (Figure 8).

Thus, comparing activity in the first five minutes of the digital and conventional conditions, it appears tool-focused work during the digital sketching condition has reduced time spent on C's moves to progresses potential solution candidates.

Figure 8 Working environment 00:00-05:00 of conventional sketching

During the fourth minute of her digital sketching protocol, C's tool-focus is clearly evident as she indicates dissatisfaction with her work out of a concern for her own abilities, "It's just a mistake. I'm not good at using this tool" (Figure 9, Mistake with tool). Dissatisfaction is also evident as C reflects upon the result of her tool-focused moves, "I think I didn't use all these tools fully" (Figure 9, Inability to use tool), followed by an attempt to locate a desired tool, "Where's the tool that I want. Maybe a little bit confused and ah..." (Figure 9, Search for correct tool). The original mistaken move plus the subsequent reflection upon an inability to utilise digital sketching and continued moves to identify a desired function appear to also tie her activities to the problem space as she continues to name sub-problems to pay attention to. 
Figure 9 Apparent attempt to stimulate design ideation, digital condition 04:00-07:00

In further work characterised by attention shifts from design to tool-focus during the digital condition, $\mathrm{C}$ attempts to develop an understanding of a more abstract concept of luxury. After repeatedly circling the word 'luxury', $\mathrm{C}$ draws and reflects upon a sketch of a woman, "And then, just I'm thinking of the image that I have. A woman wears a dress and wears the sunglasses and walks around like a model” (Figure 10).

Figure 10 Sketch of a woman, $5^{\text {th }}$ minute of digital condition

C then considers the relationship of luxury to more pragmatic requirements of use, "So, it is not connected to daily life actually." At this point, she admits again to feeling confused before shifting her focus away from establishing an understanding of luxury to consider materials, "and so thinking about materials. What materials I have to use." Although this appears to provide an opportunity to consider the relationship between materials and luxury, she is distracted as attention moves once again to the location of a particular function (Figure 10, Search for correct tool).

C's continued tool-focus shifts away from thinking about her design ideas appear to disrupt and fragment her ability to engage solution ideation. This fragmentation of design-driven activity has resulted in an inability to transition between naming important considerations in the problem space to moving to propose and develop potential solution ideas through design-focused moving and reflecting.

At the start of the digital condition's seventh minute, $\mathrm{C}$ admits to remaining confused, "Confused. What should I do? I don't know what, what direction I have to go." It is at this point that an analogue display idea frame re-emerges. However, instead of immediately moving to explore its suitability through design-focused moving and reflection, as seen in the emergence of framing events in the conventional condition, $\mathrm{C}$ returns to reaffirm already named problem considerations, underlining previously written words, “daily, use, attractiveness, resistant, scratches". As before, tool-focused activity appeared to limit C's ability to propose and explore solution ideas through transitions between problem definition as naming and solution exploration through design-focused moving activity. Her continued shifts to and from tool-focused work fragments her reflect-in-action, to be replaced with somewhat frustrated uncertainty.

Figure 11 Sketch of a previously encountered watch, $8^{\text {th }}$ minute of digital condition

In contrast, by the fifth minute of the conventional protocol, $\mathrm{C}$ has generated a sheet of sketch work, notes and schematic drawings (Figure 12). C continues her design-focused moves through sketching, notably including detail of where the watch display interfaces with the strap. From exploring the new UI frame's suitability to address the design sub-problem of display, C's attention shifts to the watch's strap, as indicated by a new frame, "Velcro I see. A sticking method. Yeah. Yeah a fixing method for Velcro" (Figure 12, first framing event). Moves made in the testing of the UI display frame have included an incidental representation of the watch's strap (Figure 12). This appears then to prompt the re-emergence of a focus upon strap design and provides the stimuli through which $\mathrm{C}$ 
reengages the strap sub-solution as Velcro emerges as a new frame worthy of exploration (Figure 12, second framing event).

It appears a lack of tool-focused activity in the conventional condition (as also indicated in the statistical analysis) has resulted in fewer transitions between activities and increased time spent in design-focused moving (Figure 12, longer yellow bars). This appeared to provide $\mathrm{C}$ with a more flowing reflective-practice, as the problem orientated naming of considerations transitions to designfocused moving to propose, reflect upon and develop conceptual ideas.

Figure 12 incidental sketch representation of watch strap, $7^{\text {th }}$ minute of conventional sketching

In contrast to the solution focused, move-driven nature of reflection-in-action observed in the conventional condition, at the digital protocol's $10^{\text {th }}$ minute C's work again shifts to a tool-focus (Figure 13, 10:00 Zooming) before indicating frustration at being unable to engage in actions taken for granted in the conventional sketching condition, "Err, it would be much better to, it would be like a sketchbook. I can change the page. It would be better" (Figure 13, Zooming, Inability to change pages).

Figure 13 Re-sizing and re-locating work, $10^{\text {th }}$ minute of digital condition

After naming time constraints and reflecting upon a resized and repositioned first page, $\mathrm{C}$ appears to become increasingly aware of how her tool-focus is implicating an ability to engage design ideation, "Maybe I'm not so good at using these tools so maybe I'm thinking about what do I have to do in the second page, I'm reminding myself and seeing and. So, I'm a little bit confused" (Figure 13, unsure what to do next). $C$ then begins to scribble onto her work surface in an apparent attempt to facilitate the stimulation of ideas, "I wanted to, wanted to draw, nothing meaningful...just drawing and thinking and refreshing my mind about what I have to do". After reflecting upon the appropriateness of a curved line, C again shifts to a tool-focus to locate desired functionality, "So I set the shape as an oval, an ellipse...so...err, I wanted to, err, change something, but it was already there, so I find it. Um...” In this section of the digital protocol it appears that the participant has achieved some awareness of the influence that her tool-focused approach is having on her ability to ideate design intentions. However, her ability to engage in design-focused moves continues to be frustrated by the fragmentation of her moving activity as she is distracted by tool-focused shifts.

In contrast, at the end of the $10^{\text {th }}$ minute of the conventional sketching protocol, $\mathrm{C}$ continues to explore the detail of the solution frames, "Yeah, the fixing method. Maybe mainly I'm thinking and considering the...fixing part as a very important issue..." After completing a sketch of the watch-strap fixing, she returns to the previous frame of a new UI display, "so I just decided to use that display", before moving to explore the frame's appropriateness through sketching. C's design-orientated ideation work continue to increase the duration of design-driven moving and reflecting activities which, in turn, provides potential opportunities for the proposition and development of solution ideas.

During the same period of the digital sketching condition, however, $\mathrm{C}$ appears to show concern over a lack of progress. It now appears increasingly difficult to break from her tool-focus, "I think the 
tools also affect the design...so...I'm just trying it" (Figure 14, Tool as influence on design). At this point, C writes across the tablet in large, purple letters the word, 'concept' (Figure 14).

Figure 14 Working environment 13:00-15:00 of digital sketching

C then attempts to change her approach as she mentions her own experience of going to the beach, "actually...err...yesterday I went to the beach, so what about setting the concept as the beach?" However, this idea is abandoned due to its inability to connect to the core concept of luxury, "but there was no relation between daily use of luxurious things". C's attention again shifts to the digital sketching tool as she moves to identify a particular function (Figure 14, Looking for graduation tool), before re-scaling the canvas and naming the importance of function identification, "and I'm thinking I have to find the graduation tool, but I can't find it" (Figure 14, Importance of graduation tool). It is at this point $\mathrm{C}$ attempts to become consciously detached from her tool-focus as she draws a large oval in the center of the canvas.

Increasing tool-focused activity in the digital condition's mid-section (Figure 14, moving, black bars) appears to have reduced the attention given to design ideation, evidenced by little in the way of solution orientated moving or reflecting activity (Figure 14, flow-chart). The implications of this for the progress of design work are a reduction in the proposition and development of solution ideas, leading to increasing participant frustration in her inability to engage in solution development.

In contrast to the tool-focused work seen in the digital condition, the same period of conventional sketching (10:00-20:00) sees C’s attention shift towards design detail, “...oh...battery, yeah...The same things, displaying it...and", before exploring the size and form of the design through a second sketch, "Um, I just wanted to find the better line that I wanted to draw". C again enters a period of design-focused moving activity, whereupon a strap concept and fixing mechanism is sketched, "And, again, I wanted to find a more fancy fixing method...So, just continue to think...” (Figure 15)

Figure 15 Exploring fixing mechanism, $17^{\text {th }}$ minute of conventional sketching protocol

As before, design-focused sketching appears to act as stimuli for the emergence of the problem frame. In contrast to more frustrated work in the digital condition, this results in longer periods of moving activity, (Figure 15, yellow bars), providing opportunities for $\mathrm{C}$ to focus attention within the solution space. By the later stages of her conventional protocol, her design orientated work provides opportunities to both refine a direction and further develop her conceptual ideas.

Figure 16 Exploration of current frame through sketching, $20^{\text {th }}$ minute of conventional sketching

In contrast, by the end of the $16^{\text {th }}$ minute of the digital protocol, C's work has become ever more tool-focused. The increasingly diminished time spent in design-orientated moving and reflecting activity is, instead, occupied by developing an abstraction relating to the analogy of luxury, "And, this is the night...of the city. And the part below should be the daytime of nature" (Figure 17). However, attention is once again distracted by a surprising colour change, "Err, I think I moved to here so it was, 
err, it was automatically changed to this, this colour, but it was, that point was in here so it was almost grey".

Figure 17 TDR-focused moving, 18:00-20:00 of digital condition

Following a brief attempt to reflect upon her sketching work in terms of design development, "So, I'm just drawing like this. Yeah", C returns to her tool-focus, naming a desire to locate functionality (Figure 17, to find fit tool 20:00). This then continues as she moves to rotate the image (Figure 17, Rotating image) before reflecting upon the results of her efforts in terms of the expression of design intent, "but then I thought it is not good for..." Her tool-focused work appears to both fragment and frustrate a desire to achieve a more solution-focused approach to the design task (Figure 17, bar-chart).

At approximately the same period in the conventional condition, $\mathrm{C}$ identifies a difficulty to know what to do as she adopts a sudden tool-focus, "so I draw it below this thing. And I keep drawing it. It will be better for it". C then announces her intention to continue to shadow and shade as a thinking aid, "So just keep shadowing and thinking and err more ideas I need yeah". At this point, C opens a pack of marker pens and proceeds to create colour swatches for each in turn before announcing the choice of colour after which she is informed that time allocated to the task has ended. What prompts this sudden change at the conclusion of the conventional protocol is unclear. However, as with the toolfocused activities seen in the digital condition, her tool-focus shift appears to result in a breaking of reflection-action flow, through design-focused moving and reflecting activity, to be replaced with less productive tool-focused moving. This then appears to course design ideation to stop and be replaced with activities with reduced cognitive load (i.e. considering starting a new page; checking colours on page, Figure 18).

Figure 18 Choosing colour variations, $24^{\text {th }}$ minute of conventional task

In the twentieth minute of the digital condition, $\mathrm{C}$ names a desire to change both her own illustration and return to locating a particular function, "So I wanted to use the graduation tool and change the colours of the sky. Morning and afternoon and evening. Something like that" (Figure 19, Desire use graduation tool). Frustration is again expressed in her inability to develop her design ideas, and concludes the protocol by re-writing key words in thick red lettering (Figure 19).

Figure 19 Working environment in final minute of digital sketching condition

The influence of a tool-focus identified in the quantitative comparison is clearly evident at the conclusion of C's digital protocol (Figure 19, black bars). Tool-focused naming (Figure 19, orange/black) and moving (Figure 19, Yellow/black) leave work towards any kind of solution idea undeveloped and lacking in any recognisable detail or direction. This also appears to have implications for her emotional state as at the end of the protocol she adds six question marks to the workspace (Figure 19). With her final few tool-focused moves of the remaining allocated time, C deposits her last expression of intent to the edge of the screen. 
C's continued shifts towards tool-focused naming and moving activity in the digital condition have dramatically impaired an ability to develop design intent through design-focused moving. Instead her work becomes fragmented and uncertain. This in turn has limited the productivity of the ideation session in the digital condition and frustrates the participant's ability to propose and progress design intentions. This is in contrast to the conventional condition, where design work is characterised by a more flowing reflection-in-action, providing greater opportunities to move between problem definition and solution ideation, characterised by increased design-driven moving and reflecting activity.

\section{Discussion \& Conclusions}

This study has explored how conventional and digital sketching influences focus of attention between tool and design-driven activity (Self, Evans et al. 2014) and implications for reflection-in-action (Schon and Wiggins 1992, Valkenburg and Dorst 1998). Design activity in digital and conventional sketching conditions was empirically compared through a retrospective think-aloud protocol analysis of conceptual design work. In contrast to existing studies of design sketching (Gero and Mc Neill 1998, Tang, Lee et al. 2011), the current investigation employed reflection-in-action as the basis of a concept-driven coding frame. In a mixed-methods approach to the examination of protocol data, a statistical analysis first compared distributions of and transitions between naming, framing, moving and reflecting activity in the two sketching conditions. Tool and design-focused activity between and within each of the four categories of activity was also examined. Statistically significant differences were further examined through a fine-grained qualitative comparison of design ideation during digital and conventional sketching.

Results showed that frequencies of tool-focused activities were significantly increased in the digital condition compared to conventional sketching. In contrast, non-tool-focused activity significantly increased during conventional sketching. These findings indicated that the participants' use of digital sketching resulted in a greater focus upon tool of design representation at the expense of design-driven work. The implications of a greater tool-focus during digital sketching appeared to be a limited number of design-driven moves, resulting in limited opportunity to transition from problem definition to the proposition and exploration of solution ideas. Increased tool-focused activity in the digital condition also resulted in what appeared to be the fragmentation of a reflection-action flow. This was instead replaced by frustration due to an inability to effectively engage conceptual design ideation during digital sketching. In contrast to the conventional sketching condition, results indicated how design-driven moving activity, as opposed tool-focused work, potentiated the generative exploration of solution ideas.

The statistical analysis also showed how the two activities of tool-focused moving and reflecting were significantly increased in the digital sketching condition. The increased tool-focused moving frequencies indicated how design work in the digital condition was interrupted by attention shifts towards the tool of design representation (digital sketching tool). This was in contrast to conventional 
sketching, where significantly increased design-driven moving activity appeared to potentiate active exploration of the solution space, resulting in what appeared to be more sustained and ultimately productive reflection-action flows. In terms of implications of significantly increased tool-focused moving in the digital condition, the qualitative comparison of protocols indicated that the act of transitioning to a focus upon the digital tool appeared to impede an ability to move into a reflective mode of working. In contrast, a design-driven focus of attention provided opportunities to propose and progress solution ideas through increased design-focused moving and reflecting activity.

The qualitative analysis also indicated how non tool-focused work supported both increased exploration of the design problem and the proposition and development of design ideas through transitions from the naming of important objects within the design situation (Schon, 1983) to the framing of sub-problems and partial solutions. These frames were then more effectively explored through sketching as both an experimental and developmental activity. That is, following framing events, design-focused sketching provided a necessary means for the development and testing of the frame's suitability to address an emerging understanding of the design problem.

The investigation provides evidence of how digital sketching can increase tool-focused activity during conceptual ideation and how this increase implicated an ability to engage generative design ideation through design-driven work. In terms of implications for reflection-in-action, if reflective practice is a conversation with the situation, then it appears tool-focus has the potential to inhibit the communication flow between designer and design ideation. That is, tool-focused activity appears to limit opportunities to adopt solution-focused strategies (Cross 2007) in pursuit of problem resolution. In contrast, reduced tool-focus, or design-driven approach, appeared to provide greater opportunities for the exploration of solution ideas (i.e. significantly increased design-focused moving and reflecting). The implications for this appeared to be a more generative and flowing reflective-practice, with a resulting increase in solution ideation.

Although the results showed differences in the participants' conceptual ideation in the two sketching conditions, caution is required in the generalisation of these findings. Whilst the sample size is similar to other protocol studies of design activity (Suwa, Purcell et al. 1998, Almendra and Christiaans 2009, Perry and Krippendorff 2013), the statistical analysis is based upon a relatively small number of participants. Moreover, although possessing a design education, internship and industrial experience, the subjects may not be described as designers at the highest level of knowledge and skills acquisition (Dreyfus and Dreyfus 1980, Lawson and Dorst 2009). Nevertheless, the results indicate how a focus on the tool of design representation appears to have profound implications for an ability to engage in moves aimed at an exploration of solution ideas, with implication for reflection-inaction in terms of a flowing conversation with the situation. That is, a tool focus appeared to fragment and frustrate the flow of ideation work to the detriment of attempts to engage in appositional bridgebuilding between problem definition and solution ideation.

Findings from the study also contrast with those of others (Jonson 2005, Tang, Lee et al. 2011), 
contributing to debate on approaches to the investigation of design activity (Dorst 1997, Vermaas and Dorst 2007). For example, Jonson's (ibid) study describes how various tools were used in support of conceptual ideation, indicating the influence of the designer over tool used. In contrast, the current study provides evidence to suggest how the tool itself may also implicate the designer's ability to engage in generative solution ideation. Likewise Tang et al's (op cit) study suggested digital sketching in fact had no influence upon conceptual sketching activity. In contrast the current study has indicated how a focus upon tool of design representation had implications for solution ideation due to its fragmentation of the reflective flow between designer and design representation. This then had implications for both problem definition and solution proposition and development.

More work is now required to further explore the influence tool-focused work may have upon conceptual design ideation. For example, how is tool-focus effected by designer expertise; in different contexts; and when tackling problems of differing degrees of complexity? What implications do skill and ability (for example sketching ability) has for tool-focused work and reflection-action flow during conceptual ideation? Examining the use and effectiveness of tools of design representation in various contexts provides opportunities to not only understand the influence of the tool but also the cognitive strategies employed by designers during conceptual design ideation. If it is possible to reach this level of understanding, the potential exists to develop theory that will underpin the development of, for example, more effective conceptual design tools, educational approaches, methods and strategies for the benefit of both design pedagogy and practice.

\section{Aknowledgements}

We would like to thank Seong Geun Lee and Seongryong Kim for their valuable research assistance towards this project. This work was supported by the year of 2013/14 Research Fund of the Ulsan National Institute of Science and Technology (UNIST), project number 1.120072.01.

\section{References}

Almendra, R. A. and H. Christiaans (2009) Decision making in the conceptual design phases: a comparative study. Journal of Design Research 8(1): 1-22.

Bar-Eli, S. (2013) Sketching profiles: Awareness to individual differences in sketching as a means of enhancing design solution development. Design Studies 34(4): 472-493.

Bilda, Z. and H. Demirkan (2003) An insight on designers' sketching activities in traditional versus digital media. Design Studies 24(1): 27-50.

Bilda, Z., J. S. Gero and T. Purcell (2006) To sketch or not to sketch? That is the question. Design Studies 27(5): 587-613.

Bouchard, C., A. Aoussat and R. Duchamp (2006) Role of sketching in conceptual design of car styling. Journal of Design Research 5(1): 116-148.

Chai, K.-H. and X. Xiao (2012) Understanding design research: A bibliometric analysis of Design Studies (1996-2010). Design Studies 33(1): 24-43. 
Chandler, D. (2002) Semiotics the Basics. London, Routledge.

CROSS, N. (1990) The Nature and Nurture of Design Ability. Design Studies 11(3): 127-140.

Cross, N. (2007) Designerly Ways of Knowing. Basel, Birkhauser.

Cross, N., H. Christiaans and K. Dorst (1996) Analysing Design Activity. Chichester, John Wiley \& Sons.

Do, E. Y., Gross, M. D. et al (2000) Intentions in and relations among design drawings. Design Studies 21(5): 483-503.

Dorst, K. (1997) Describing Design: A comparison of paradigms. Ph.D, TU Delft

Dorst, K. (2006) Design Problems and Design Paradoxes. Design Issues 22(3): 4-17.

Dorst, K. (2011) The core of ‘design thinking’ and its application. Design Studies 32(6): 521-532.

Dorst, K. and J. Dijkhuis (1995) Comparing paradigms for describing design activity. Design Studies 16(2): 261274.

Dreyfus, S. and H. Dreyfus (1980) A five-stage Model of the Mental Activities Involved in Directed Skill Acquisition, University of California, Berkeley.

Gero and T. Mc Neill (1998) An approach to the analysis of design protocols. Design Studies 19(1): 21-61.

Gero, J. and U. Kannengiesser (2008) An ontological account of Donald Schön's reflection in designing. International Journal of Design Sciences and Technology 15(2): 77-90.

Gero, J. S. and T. Mc Neill (1998) An approach to the analysis of design protocols. Design Studies 19(1): 21-61. Goel, V. (1995) Sketches of Thought. London, MIT Press.

Goldschmidt, G. (1991) The dialectics of sketching. Creativity Research Journal 4(2): 123-143.

Goldschmidt, G. (1994) On visual design thinking: the vis kids of architecture. Design studies 15(2): 158-174.

Goldschmidt, G. (1997) Capturing indeterminism: representation in the design problem space. Design Studies 18(4): 441-455.

Goldschmidt, G. and M. Smolkov (2006) Variances in the impact of visual stimuli on design problem solving performance. Design Studies 27(5): 549-569.

Goldschmidt, G. and M. Weil (1998) Contents and structure in design reasoning. Design Issues 14(3): 85.

Jiang, H. a. Y., C. (2009) Protocol analysis in design research: a review. Relevence and rigour: IASDR09. Seoul, Korea, Korea Society of Design Science.

Jonson, B. (2005) Design ideation: the conceptual sketch in the digital age. Design Studies 26(6): 613-624.

Kim, S., S. Jung and J. Self (2013) Investigating Design Representation: Implications for an Understanding of Design Practice. IASDR13 Consilience and Innovation in Design, Tokyo, IASDR.

Lawson, B. (2004) What Designers Know. Oxford, Architectural Press.

Lawson, B. and K. Dorst (2009) Expertise in Design. Design Expertise. Oxford, Architectural Press: 81-112.

McGown, A., G. Green and P. A. Rodgers (1998) Visible ideas: information patterns of conceptual sketch activity. Design Studies 19(4): 431-453.

Menezes, A. and B. Lawson (2006) How designers perceive sketches. Design Studies 27(5): 571-585.

Michel, R. E. (2007) Design Research Now. Berlin, Birkhäuser Verlag AG.

Nelson, G. and E. Stolterman (2012) The Design Way: Intentional change in an unpredictable world. London, MIT Press.

Pei, E., R. Campbell and M. Evans (2008) Building a Common Ground: The Use of Design Representation Cards for Enhancing Collaboration between Industrial Designers and Engineering Designers. DRS2008 Undisciplined. Sheffield, UK, Design Resaerch Society.

Pei, E., M. Evans and I. Campbell (2011) A Taxonomic Classification of Visual Design Representations Used by Industrial Designers and Engineering Designers. The Design Journal 14(1): 64-91.

Perry, G. T. and K. Krippendorff (2013) On the reliability of identifying design moves in protocol analysis. Design Studies_23(4). 
Rittel, H. and M. Webber (1973) Dilemmas in a General Theory of Planning. Policy Sciences 4: 155-169. Schon and G. Wiggins (1992). "Kinds of seeing and their functions in designing. Design Studies 13(2): 135-156.

Schon, D. (1983) The Reflective Practitioner. London, Ashgate.

Schon, D. (1987) Educating the Reflective Practitioner. New York, Jossey-Bass.

Schreier, M. (2012) Qualitative Content Analysis in Practice. London, SAGE Publications.

Self, J., M. Evans and H. Dalke (2014) Design Activity Perceptions and Performance: Investigating the relationship between expertise and practice. The Design Journal 17(3).

Self, J., M. Evans and H. Dalke (2014) The Influence of Expertise upon the Designer's Approach to Studio Practice and Tool Use. The Design Journal 17(2): 169-193.

Simon, H. (1996) The Science of the Artificial. London, MIT Press.

Someren, M., Y. Barnard and J. Sandberg (1994) The Think Aloud Method. London, Academic Press.

Stolterman, E. (2008) The Nature of Design Practice and Implications for Interaction Design Research. International Journal of Design 2(1): 55-65.

Stolterman, E., J. McAtee, D. Royer and S. Thandpani (2008). Designerly Tools. DRS2008 Undisciplined. Sheffield Hallam University

Stones, C. and T. Cassidy (2007) Comparing synthesis strategies of novice graphic designers using digital and traditional design tools. Design Studies 28(1): 59-72.

Stones, C. and T. Cassidy (2010) Seeing and discovering: how do student designers reinterpret sketches and digital marks during graphic design ideation? Design Studies 31(5): 439-460.

Suwa, M., T. Purcell and J. Gero (1998) Macroscopic analysis of design processes based on a scheme for coding designers' cognitive actions. Design Studies 19(4): 455-483.

Tang, H. H., Y. Y. Lee and J. S. Gero (2011). "Comparing collaborative co-located and distributed design processes in digital and traditional sketching environments: A protocol study using the function-behaviourstructure coding scheme. Design Studies 32(1): 1-29.

Tovey, M. and J. Owen (2000) Sketching and direct CAD modelling in automotive design. Design Studies 21(6): 569-588.

Ulrich, K. and E. Eppinger (2012) Product Design and Development. New York, McGraw-Hill Education.

Valkenburg, R. and K. Dorst (1998) The reflective practice of design teams. Design Studies 19(3): 249-271.

Vermaas, P. E. and K. Dorst (2007) On the conceptual framework of John Gero's FBS-model and the prescriptive aims of design methodology. Design Studies 28(2): 133-157.

Won, P. H. (2001) The comparison between visual thinking using computer and conventional media in the concept generation stages of design. Automation in Construction 10(3): 319-325.

\section{Appendix}

\section{Figures}




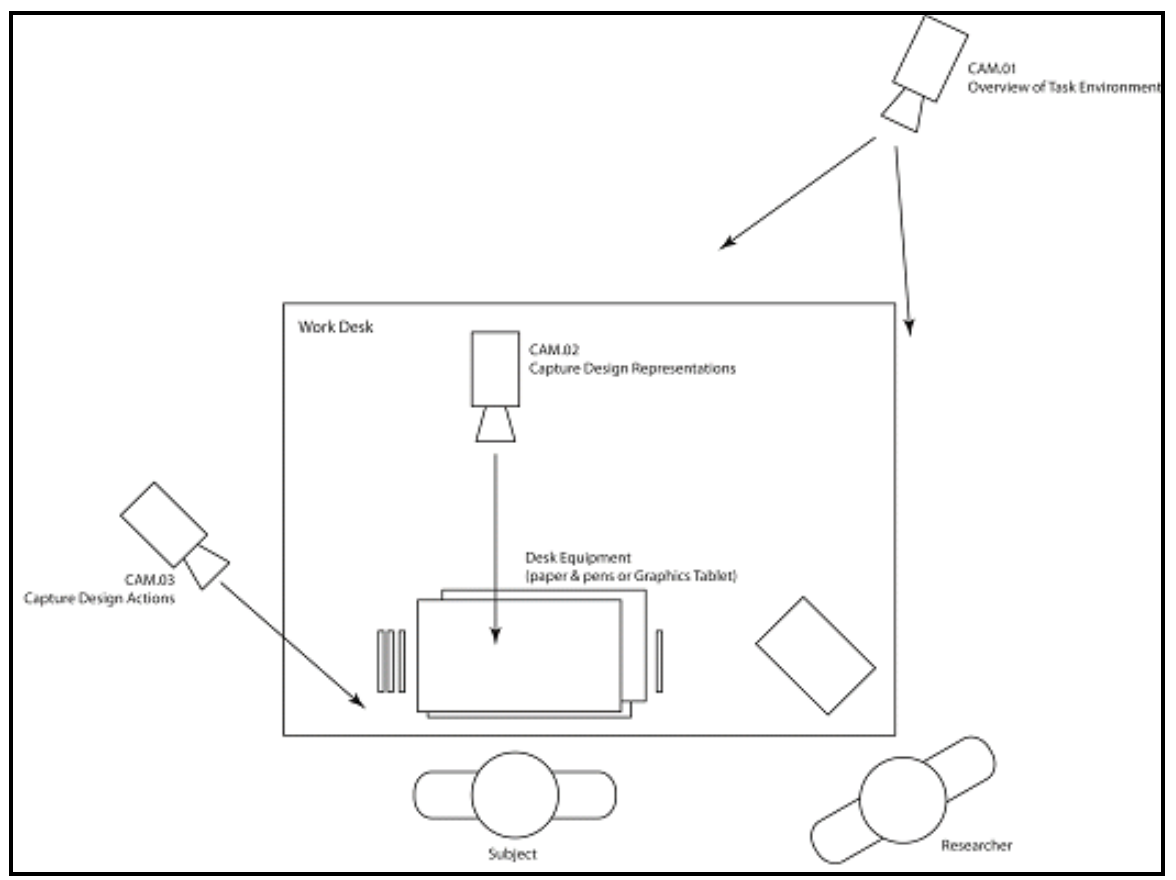

Figure 12 Task environment

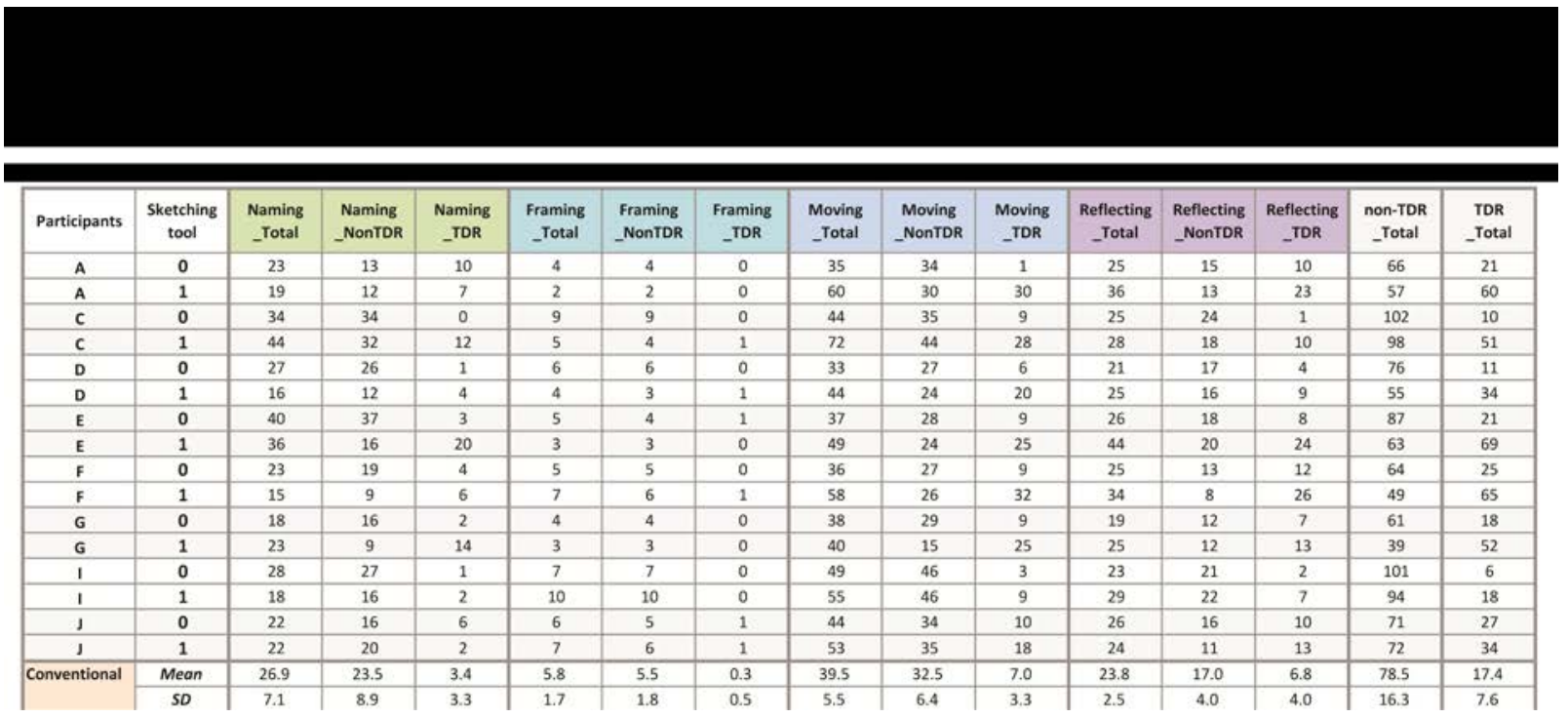

Figure 13 Frequencies of absolute encoding (f) for all participants (n) across coding frame dimensions
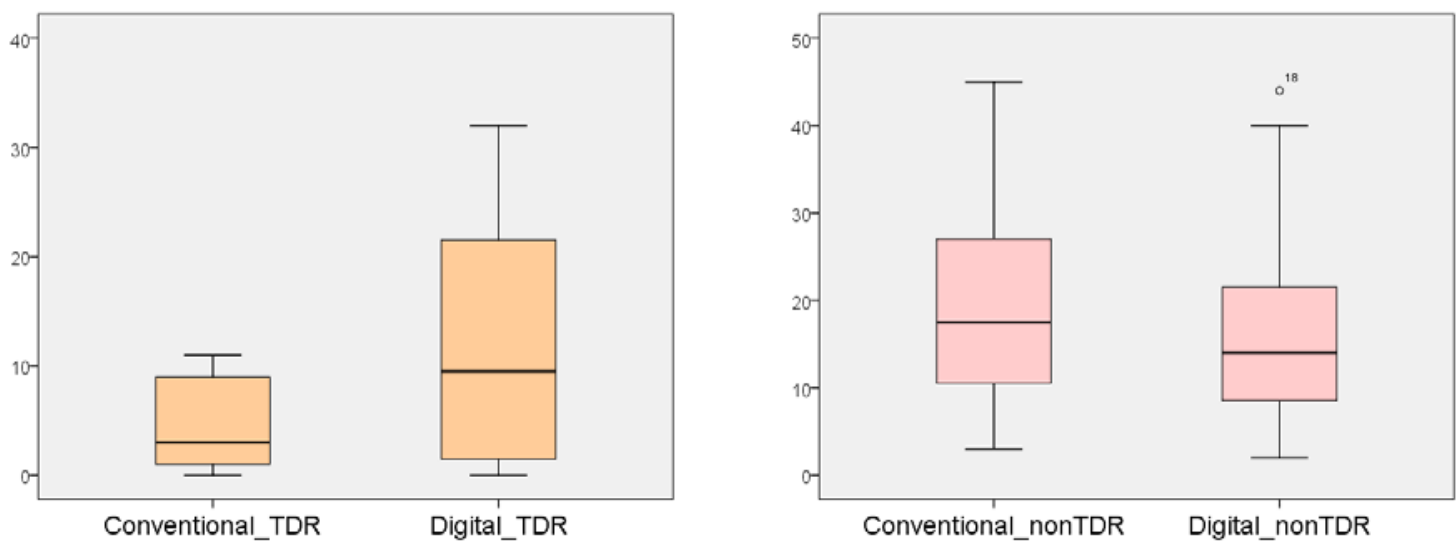
Figure 14 Frequencies of TDR and non-TDR focused activities between conventional and digital sketching.

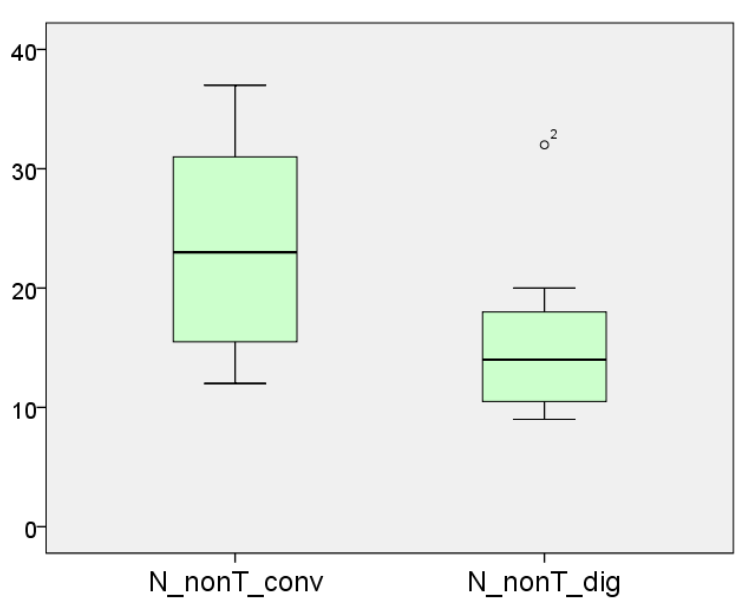

Non-TDR focused Naming

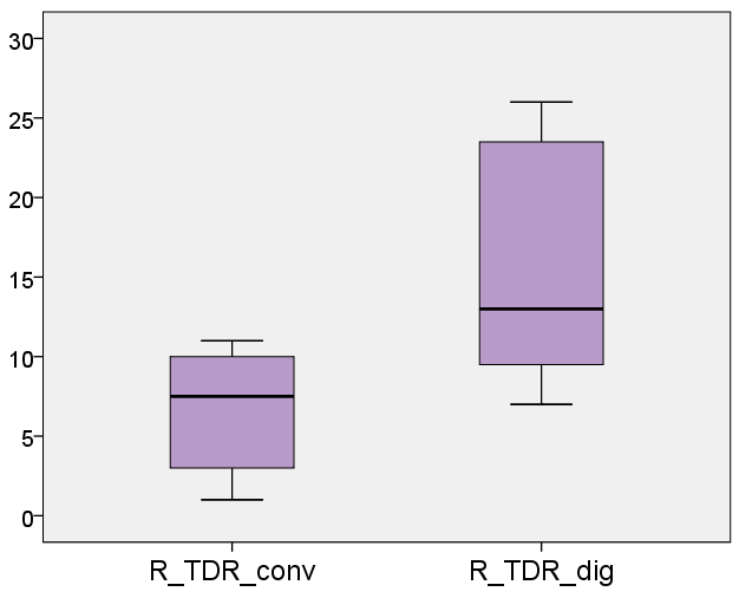

TDR-focused Reflecting

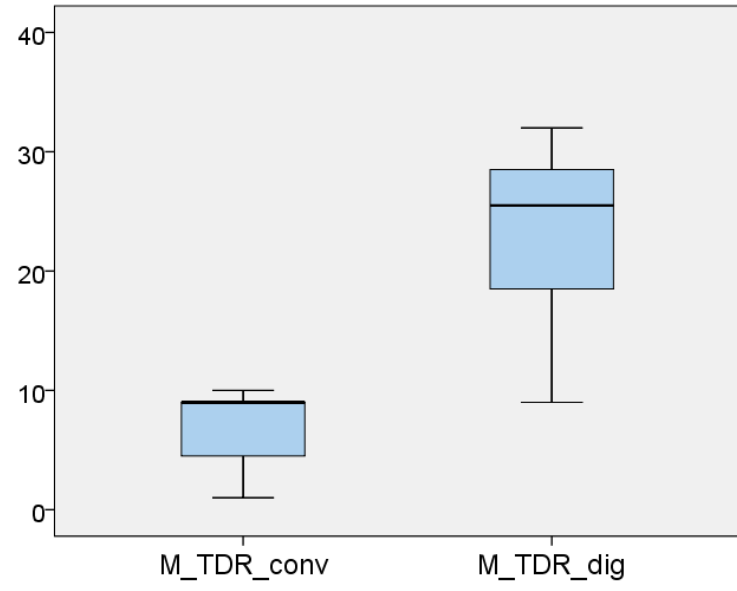

TDR-focused Moving

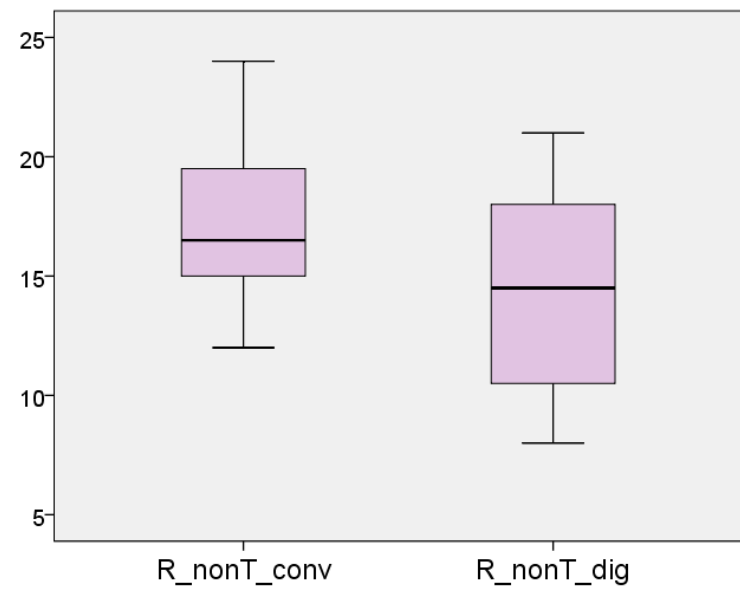

non-TDR focused Reflecting

Figure 15 Box plots of frequencies of four activities showing significant difference between conventional and digital sketching

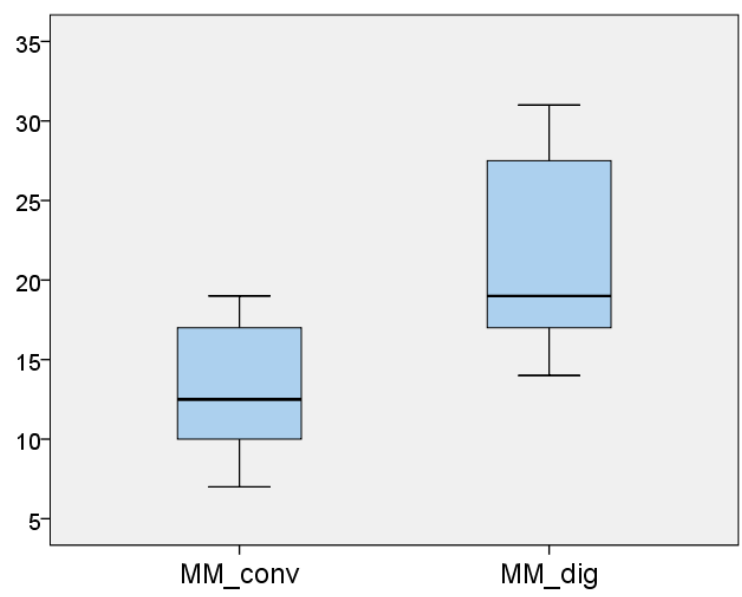

Naming to Framing (N-F)

Moving to Moving (M-M) 


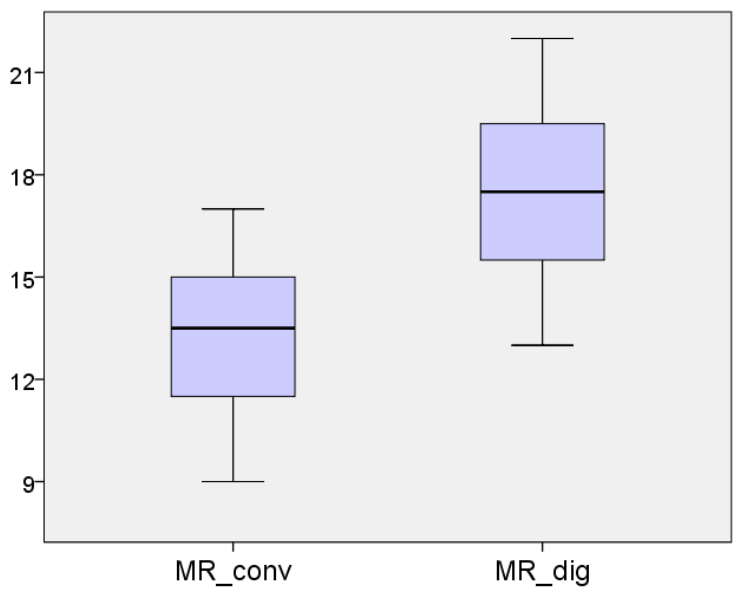

Moving to Reflecting (M-R)

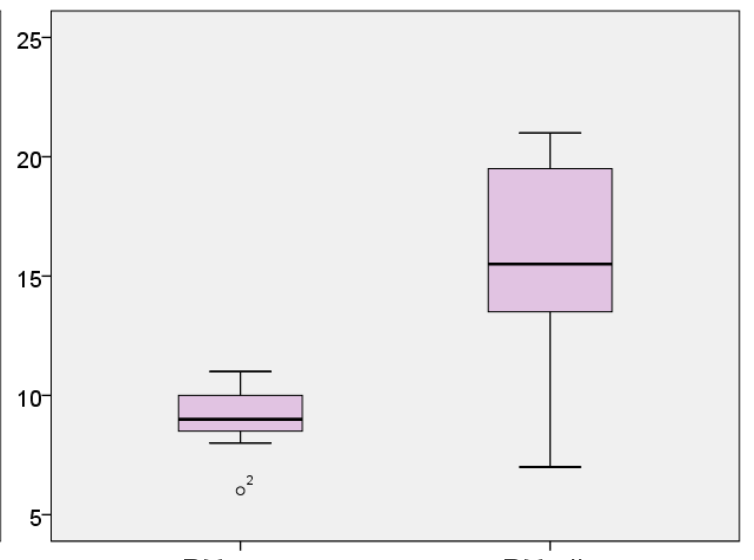

RM_conv

Reflecting to Moving (R-M)

Figure 16 Box plots of transitions showing significant differences for conventional and digital sketching. 


\section{Conventional Sketching}

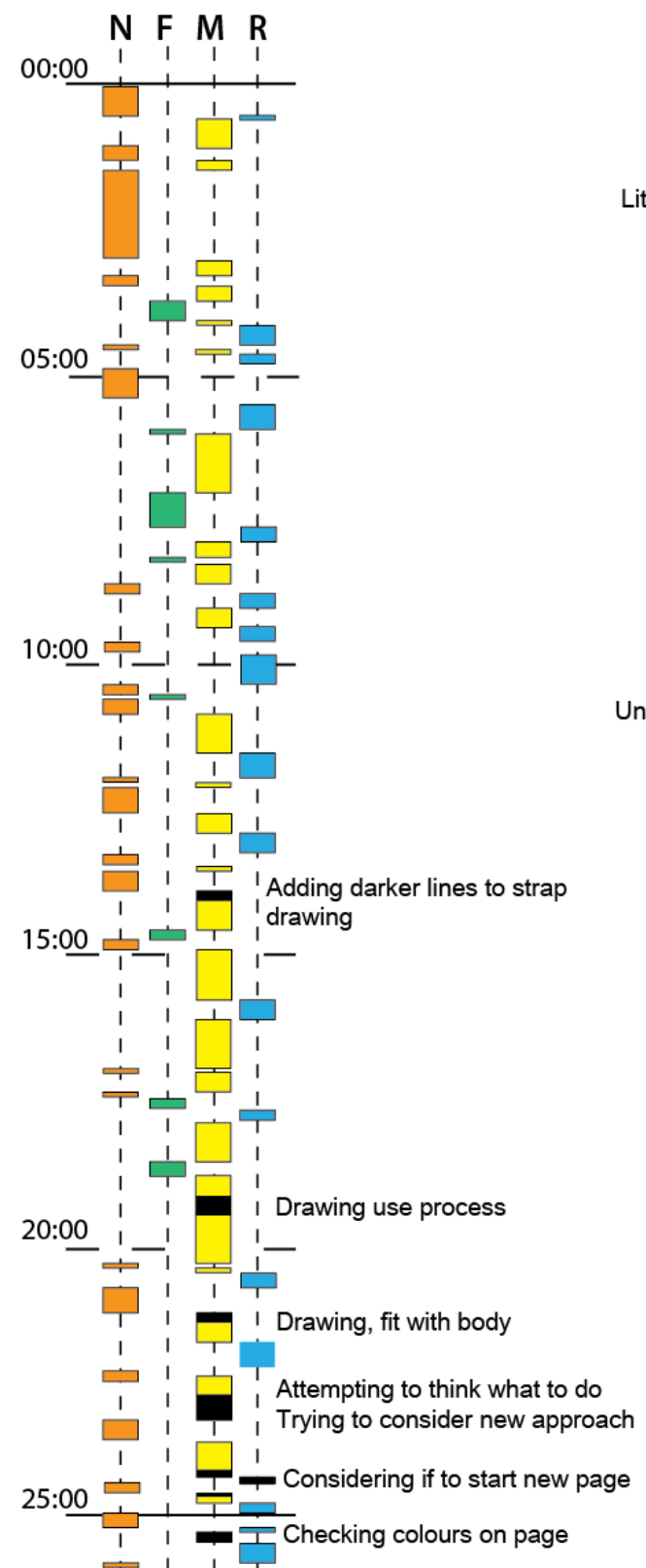

\section{Digital Sketching}

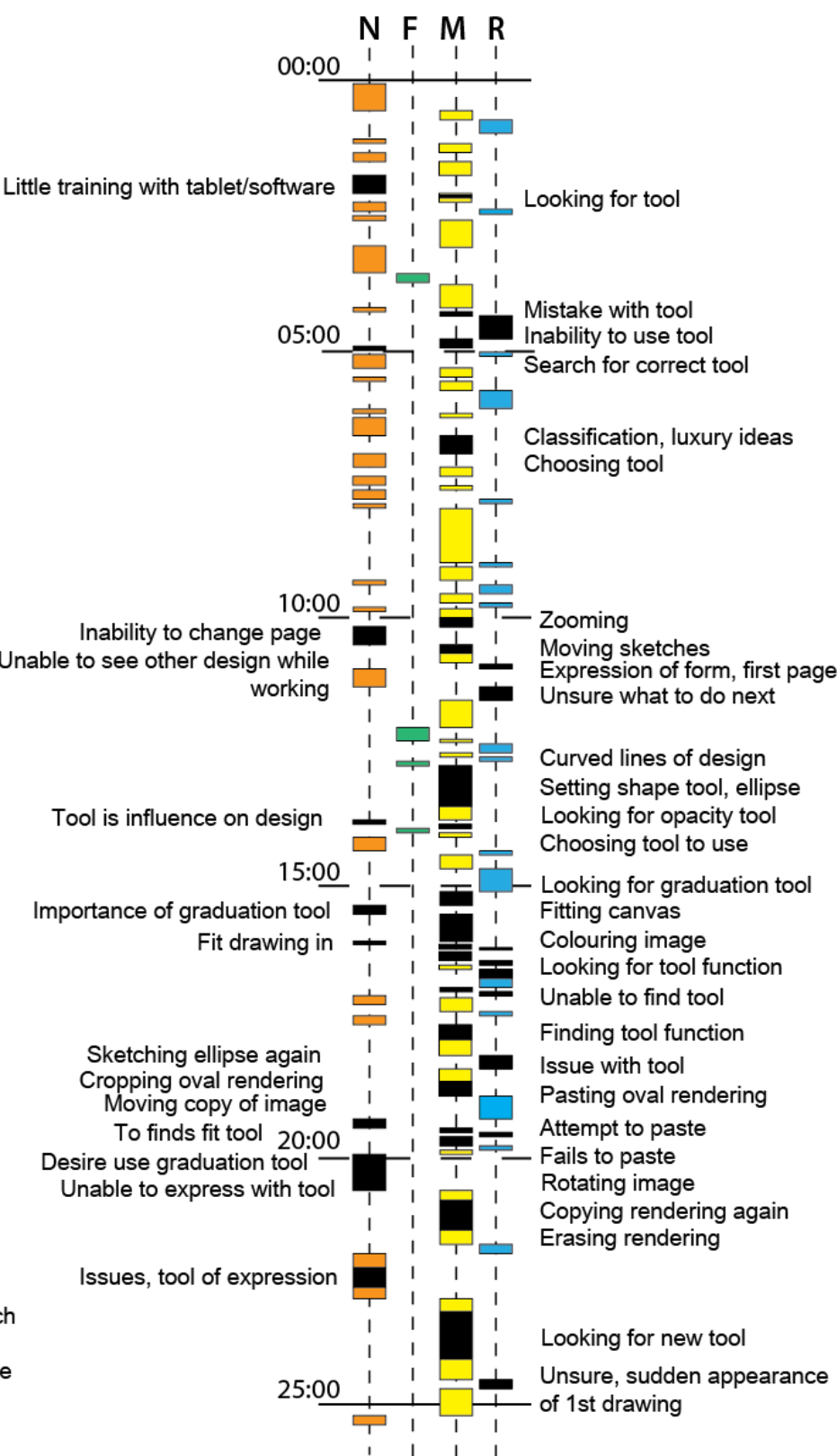

Figure 17 Flow-diagram illustrating design activity of participant C. TDR-focused activity in black 

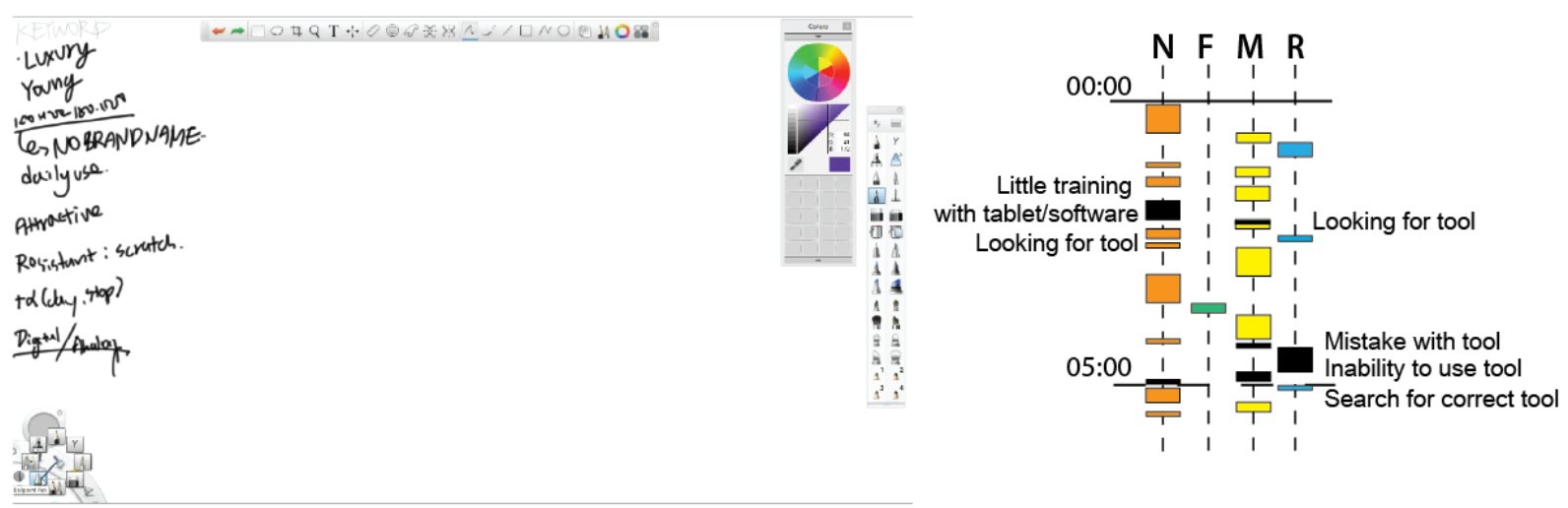

Figure 18 Screen-capture of working environment, 00:00-05:00 of digital condition
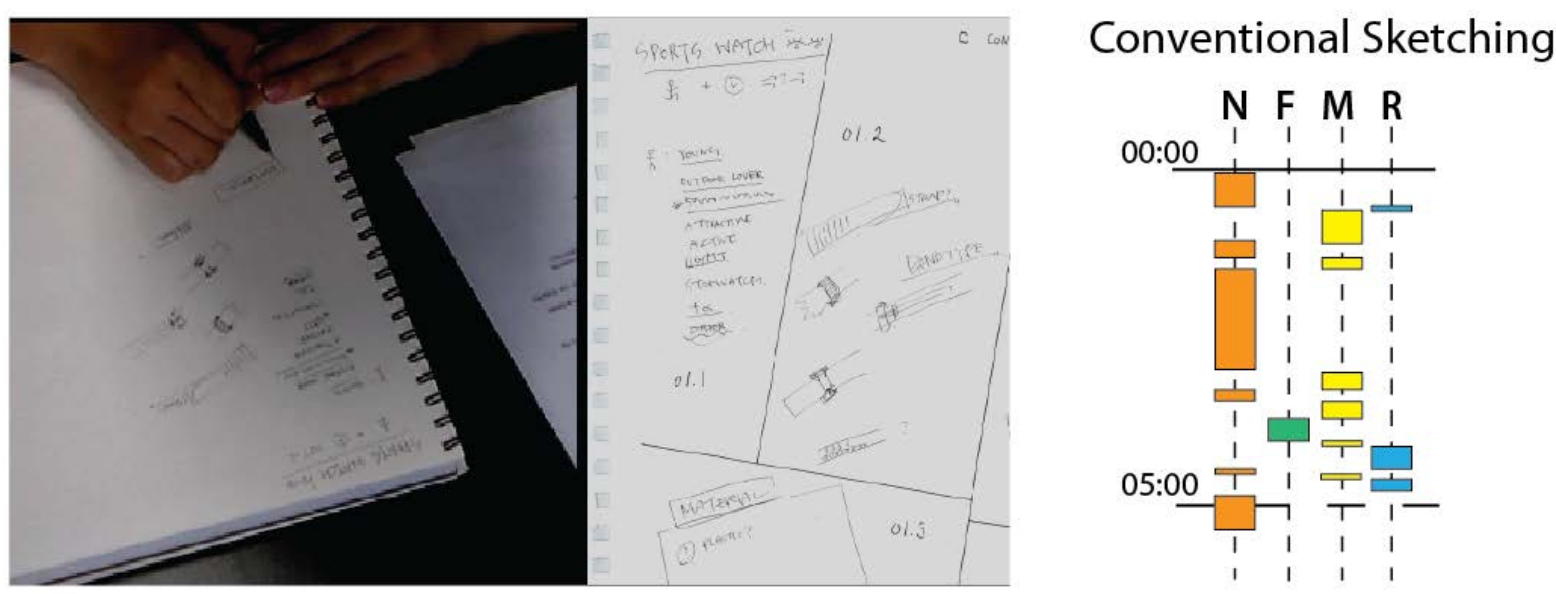

Figure 19 Working environment 00:00-05:00 of conventional sketching
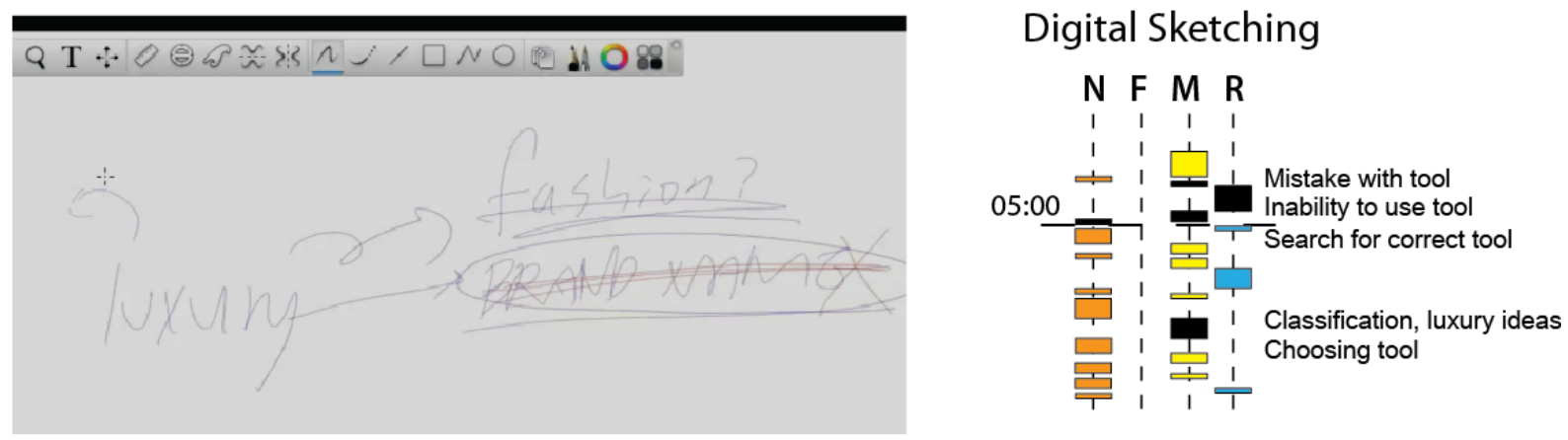

Figure 20 Attempts to stimulate design thinking, digital condition 04:00-07:00 

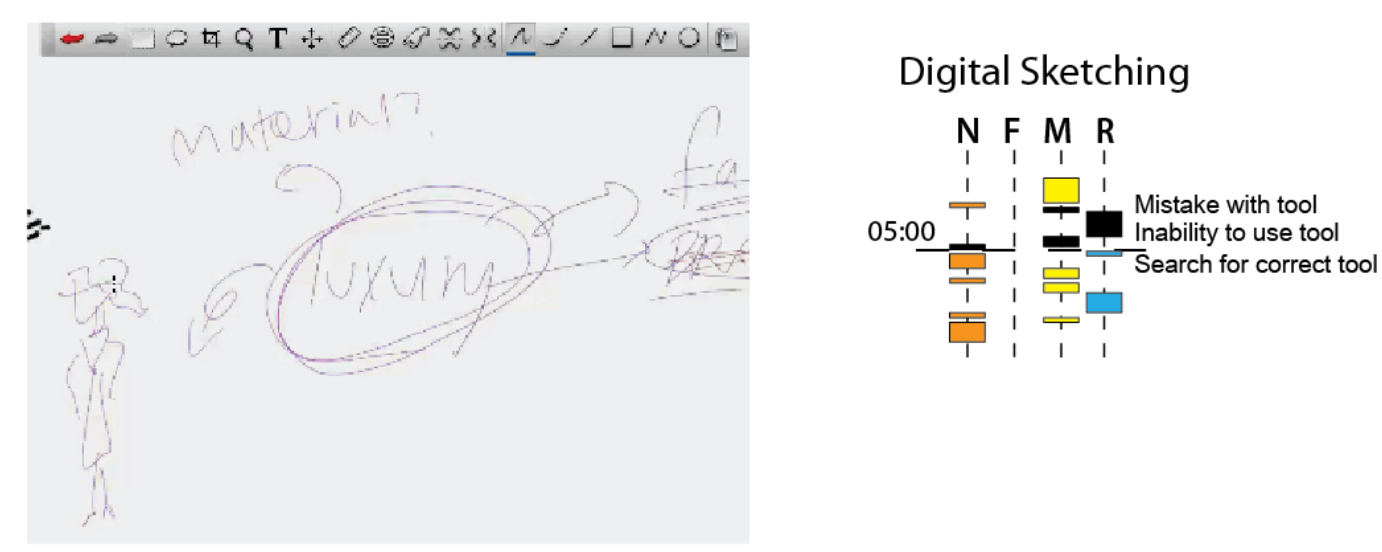

Figure 21 Sketch of a woman, $5^{\text {th }}$ minute of digital condition
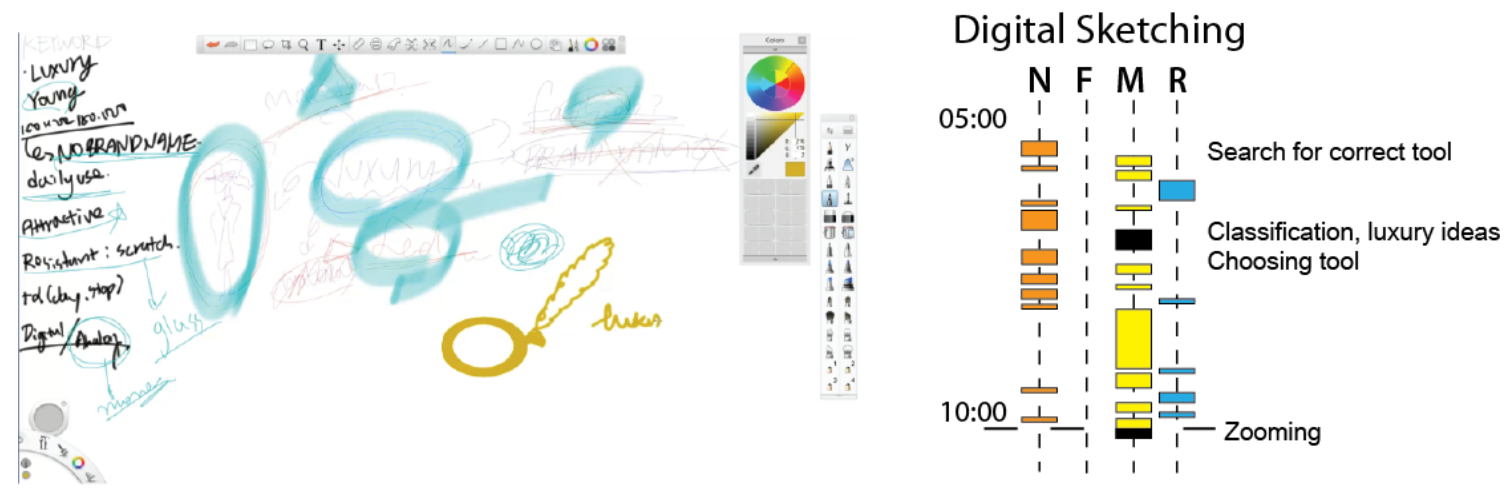

Figure 22 Sketch of a previously encountered watch, $8^{\text {th }}$ minute of digital condition
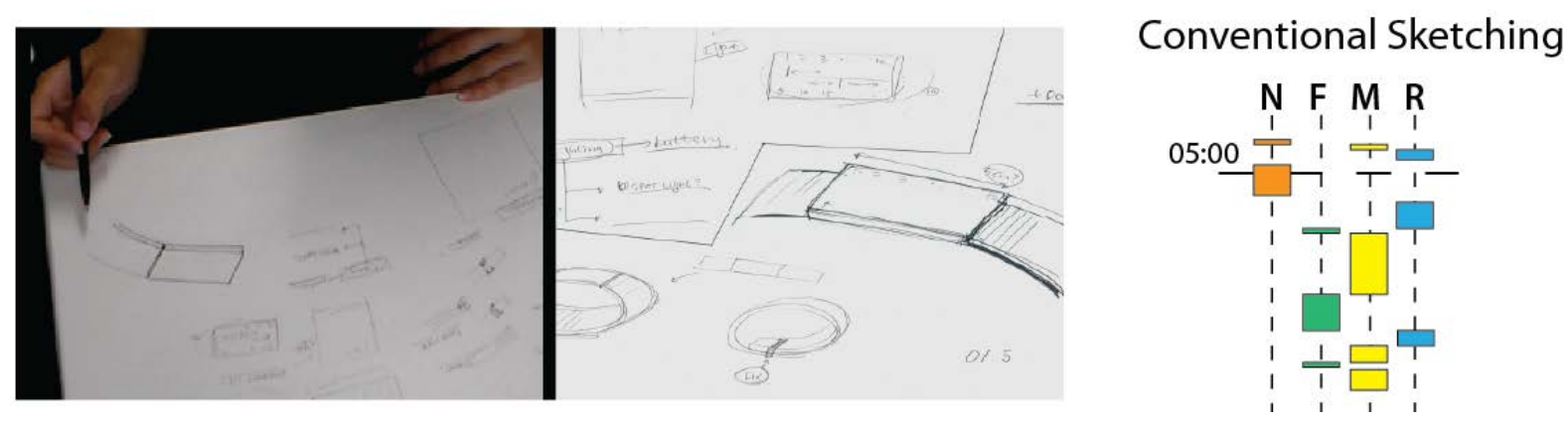

Figure 12 incidental sketch representation of watch strap, $7^{\text {th }}$ minute of conventional sketching
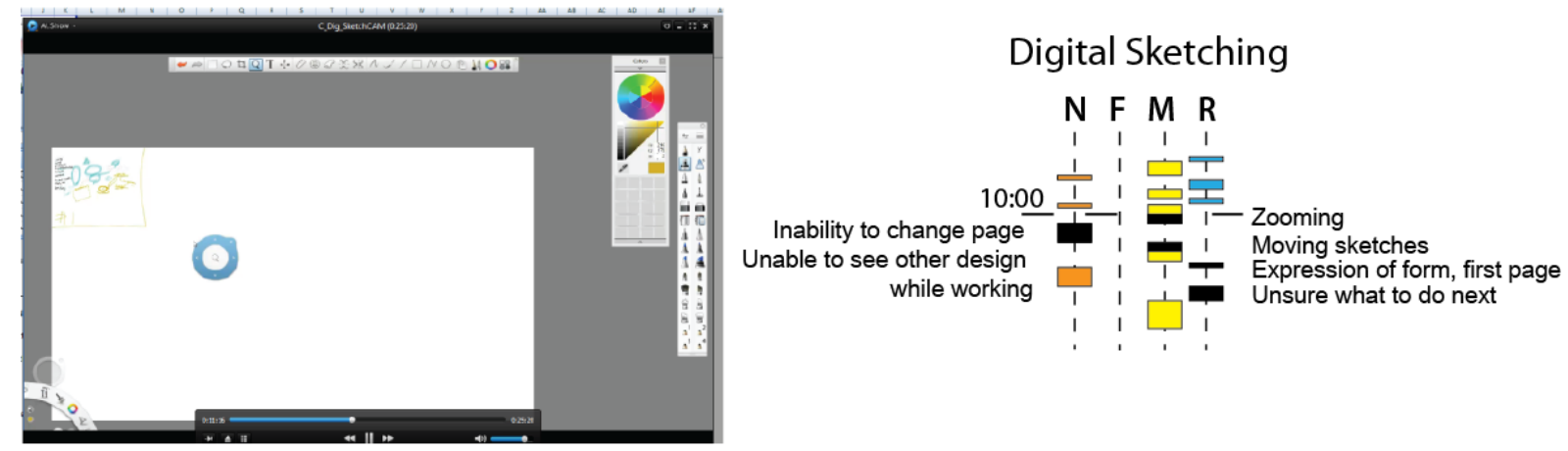

Figure 13 Re-sizing and re-locating work, $10^{\text {th }}$ minute of digital condition 

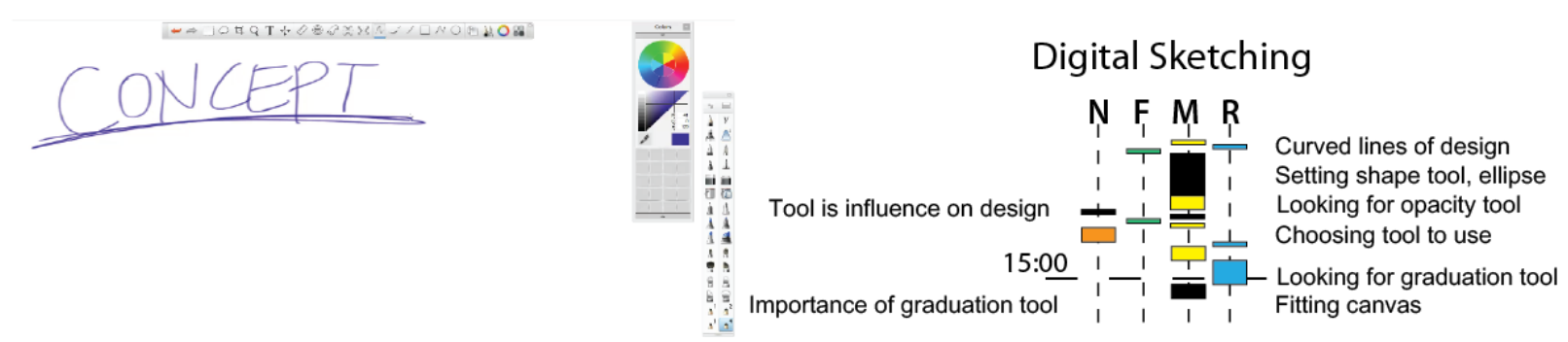
$\stackrel{i \pi}{i} e^{2}$

Figure 14 Working environment 13:00-15:00 of digital sketching
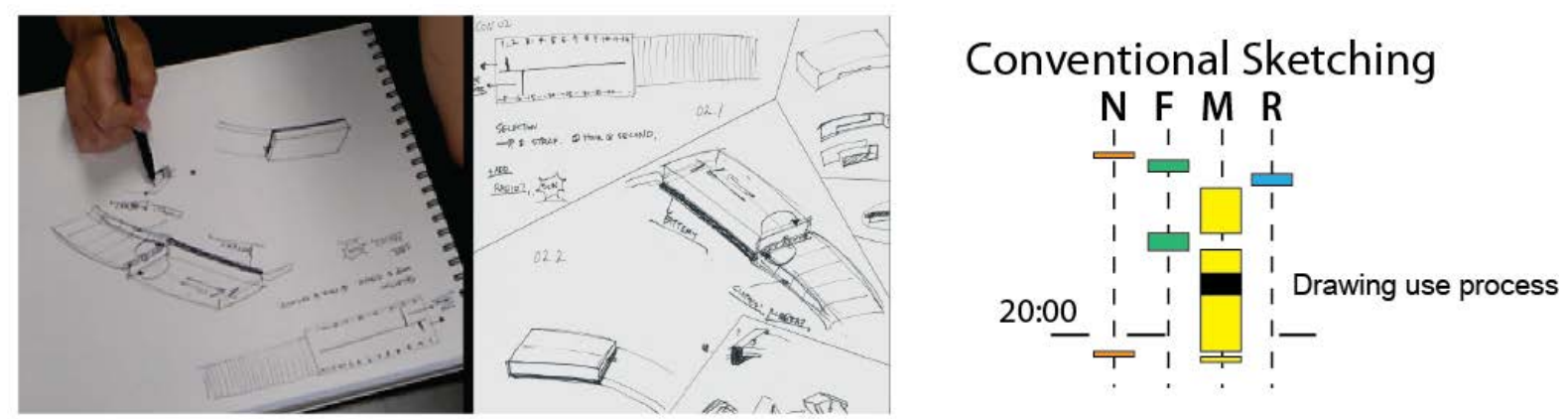

Figure 15 Exploring fixing mechanism, $17^{\text {th }}$ minute of conventional sketching protocol

\section{Conventional Sketching}
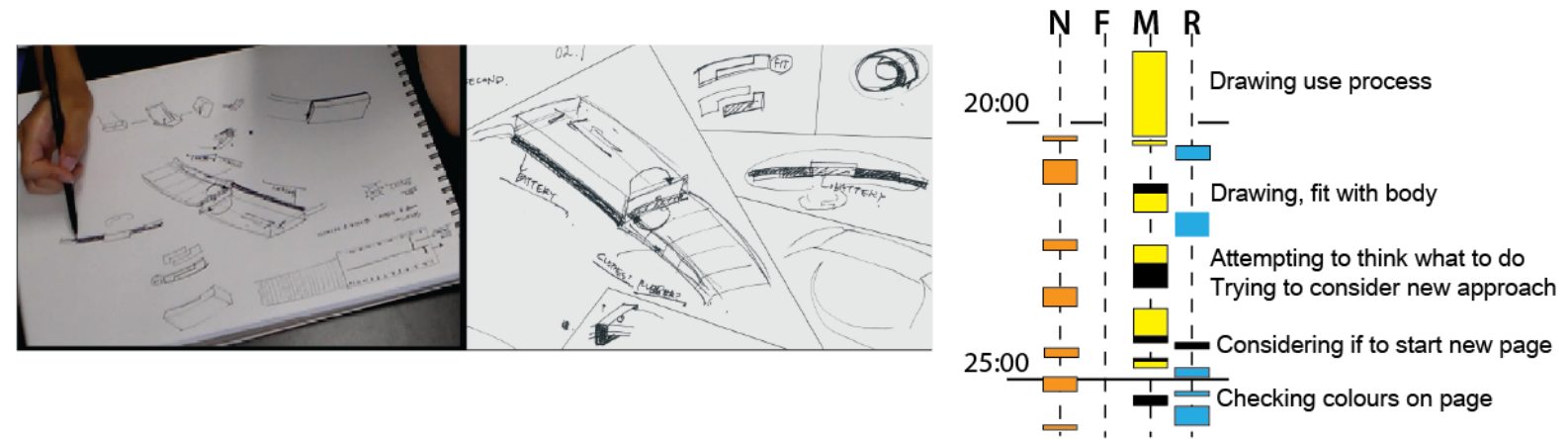

Figure 16 Exploration of current frame through sketching, $20^{\text {th }}$ minute of conventional sketching
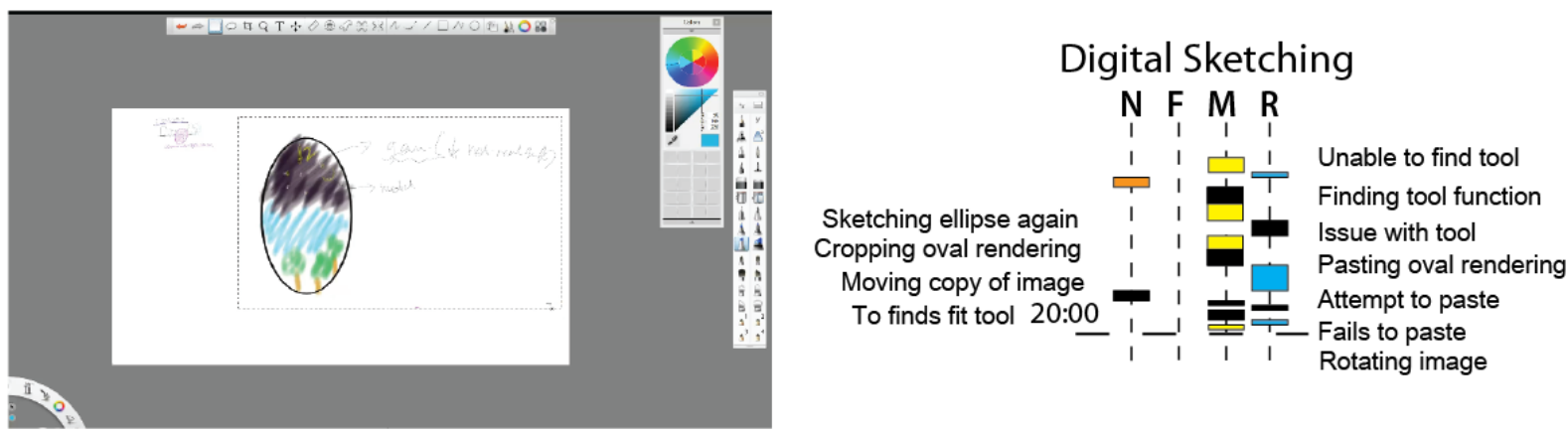

Figure 17 TDR-focused moving, 18:00-20:00 of digital condition 

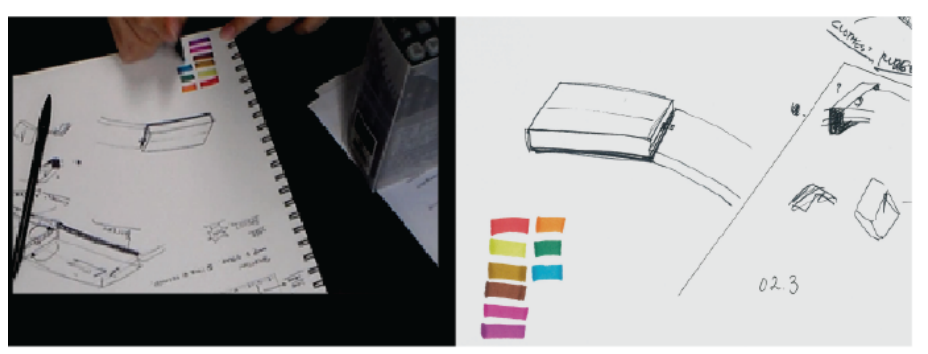

\section{Conventional Sketching}

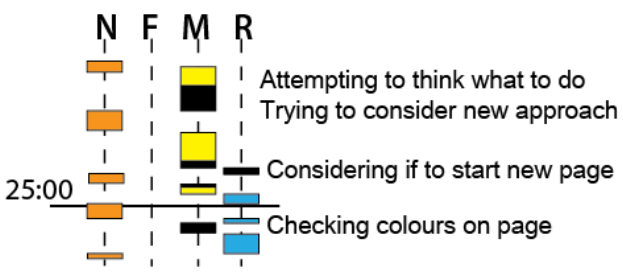

Figure 18 Choosing colour variations, $24^{\text {th }}$ minute of conventional task
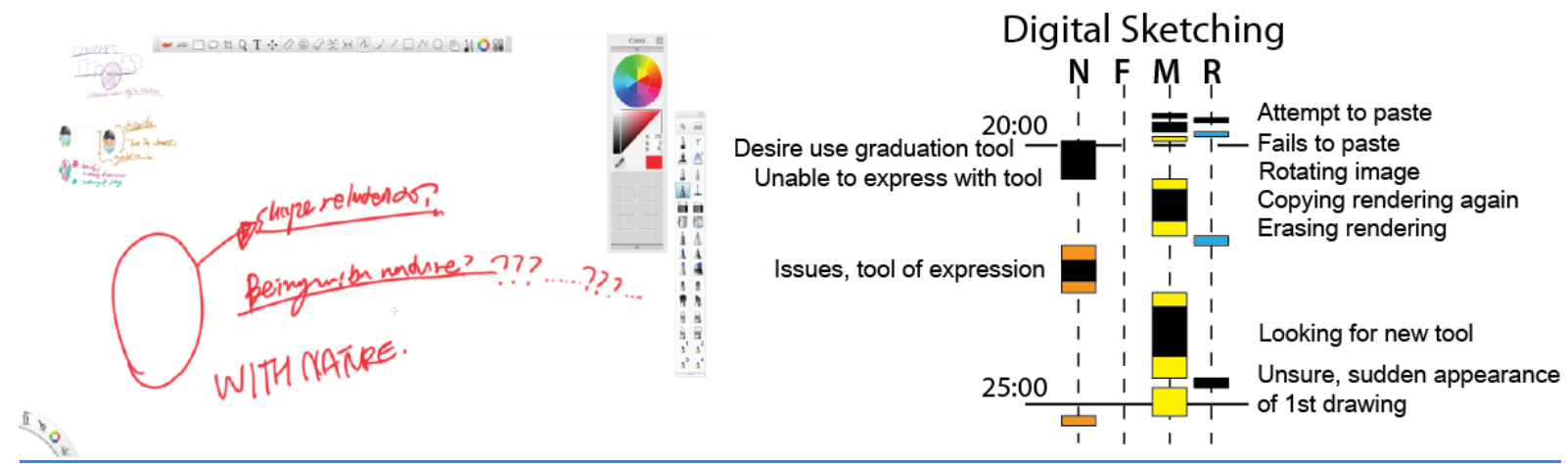

Figure 19 Work environment in final minute of digital sketching condition

\section{Tables}

Table 7 Attributes of sample of competent designers

\begin{tabular}{llll}
\hline \multicolumn{1}{c}{ Knowledge } & Coping with Complexity & Perception of Context \\
\hline Novice Designer & $\begin{array}{l}\text { Minimal or 'textbook' } \\
\text { knowledge without } \\
\text { connecting it to practice }\end{array}$ & $\begin{array}{l}\text { Little or no conception of } \\
\text { dealing with complexity }\end{array}$ & $\begin{array}{l}\text { Tends to see actions in } \\
\text { isolation }\end{array}$ \\
Competent Designer* & $\begin{array}{l}\text { Good working and } \\
\text { background knowledge of } \\
\text { area of practice }\end{array}$ & $\begin{array}{l}\text { Copes with complex } \\
\text { situations through } \\
\text { deliberate analysis and } \\
\text { planning }\end{array}$ & $\begin{array}{l}\text { Sees actions at least partly in } \\
\text { terms of longer-term goals }\end{array}$ \\
Expert Designer & $\begin{array}{l}\text { Authoritative knowledge of } \\
\text { discipline and deep tacit } \\
\text { understanding across area of } \\
\text { practice }\end{array}$ & $\begin{array}{l}\text { Holistic grasp of complex } \\
\text { situations and moves between } \\
\text { intuitive and analytical } \\
\text { approaches with ease }\end{array}$ & $\begin{array}{l}\text { Sees overall 'picture' and } \\
\text { alternative approaches; vision } \\
\text { of what may be possible }\end{array}$ \\
\hline
\end{tabular}

Sample, $n=10$

Table 8 Four conceptual coding categories

\begin{tabular}{cl}
\hline Construct & Description \\
\hline $\begin{array}{c}\text { Naming } \\
\text { (problem } \\
\text { analysis) }\end{array}$ & $\begin{array}{l}\text { When the designer is explicitly pointing to parts of the design task as being important, we code } \\
\text { the activity as naming. During naming-activity the designer is looking for relevant objects in }\end{array}$ \\
the design task. The objects to be considered in the design situation are selected and named.
\end{tabular}


When the designer frames a sub-problem or partial-solution to explore further on, then we code

Framing (synthesis) (solution simulation)

Reflecting (evaluation) this context as a frame. The frame is a context for following activities; something to hold on to and to focus on while designing. The essence was the context for further activities. The activity of naming entities is put into context through framing, and an overall perspective on the design task is constructed.

Experimental actions like generating ideas, making an inventory, sorting information, combining ideas, or comparing concepts are coded as moving. During moving activity the designer not only tries to solve the sub-problem, but at the same time also explores the suitability of the frame. The move is always characterized by a verb, identifying the activity, complemented with the content of the activity. The designer takes an experimental action based on the naming and framing of the design task.

An explicit reflection on earlier activities to know what to do next is coded as reflecting. The reflecting activity contains a critical reflection of the designer on their earlier actions. Reflections on earlier actions lead to satisfaction, the making of new moves, or the reframing of the problem. Reflection may also lead to a complete reconsideration of the designer's view of the design task, causing the designer to start naming new entities in the design situation.

Table 9 TDR (Tool of Design Representation) coding category

\begin{tabular}{|c|c|c|c|c|c|c|c|c|}
\hline Construct & \multicolumn{8}{|c|}{ Description } \\
\hline \multirow[t]{3}{*}{ TDR-Focused } & \multicolumn{8}{|c|}{$\begin{array}{l}\text { When the designer's focus of attention is on the tool through which design ideas are explored, } \\
\text { developed and communicated we call this a } \mathbf{T D R} \text {-focus. Those instances where the designer } \\
\text { consciously appears to consider the } \mathbf{T D R} \text {, its use and role in the act of designing. }\end{array}$} \\
\hline & \multicolumn{2}{|c|}{ Naming } & \multicolumn{2}{|c|}{ Framing } & \multicolumn{2}{|c|}{ Moving } & \multicolumn{2}{|c|}{ Reflecting } \\
\hline & TDR & nonTDR & TDR & nonTDR & TDR & nonTDR & TDR & nonTDR \\
\hline$Z$ value & 1.33 & -1.96 & 1.00 & -0.57 & 2.52 & -1.55 & 2.52 & -2.21 \\
\hline Sig. & .18 & .05 & .32 & .57 & .01 & .12 & .01 & .03 \\
\hline
\end{tabular}

Table 11 Numbers of transitions in 8 participants for conventional and digital sketching

\begin{tabular}{|c|c|c|c|c|c|c|c|c|c|c|c|c|c|c|c|c|c|}
\hline \multirow[b]{2}{*}{ From } & \multirow[b]{2}{*}{ To } & \multicolumn{2}{|c|}{ A } & \multicolumn{2}{|c|}{ C } & \multicolumn{2}{|c|}{$D$} & \multicolumn{2}{|c|}{ E } & \multicolumn{2}{|c|}{$F$} & \multicolumn{2}{|c|}{$G$} & \multicolumn{2}{|c|}{$I$} & \multicolumn{2}{|c|}{$J$} \\
\hline & & Con & Dig & Con & Dig & Con & Dig & Con & Dig & Con & Dig & Con & Dig & Con & Dig & Con & Dig \\
\hline \multirow{4}{*}{ Naming } & Naming & 4 & 5 & 11 & 13 & 6 & 4 & 16 & 10 & 10 & 3 & 4 & 5 & 7 & 3 & 3 & 9 \\
\hline & Framing & 2 & 1 & 2 & 1 & 3 & 0 & 2 & 1 & 1 & 1 & 3 & 1 & 3 & 1 & 0 & 0 \\
\hline & Moving & 12 & 8 & 15 & 23 & 14 & 8 & 14 & 11 & 9 & 6 & 10 & 13 & 15 & 12 & 16 & 11 \\
\hline & Reflecting & 4 & 6 & 6 & 6 & 4 & 3 & 7 & 12 & 4 & 5 & 1 & 3 & 2 & 2 & 2 & 1 \\
\hline \multirow{4}{*}{ Framing } & Naming & 0 & 0 & 2 & 0 & 1 & 1 & 1 & 1 & 0 & 1 & 0 & 1 & 0 & 1 & 0 & 1 \\
\hline & Framing & 0 & 0 & 1 & 1 & 0 & 0 & 0 & 0 & 2 & 1 & 0 & 0 & 0 & 0 & 0 & 1 \\
\hline & Moving & 4 & 2 & 4 & 4 & 2 & 3 & 2 & 2 & 3 & 3 & 4 & 1 & 7 & 5 & 2 & 4 \\
\hline & Reflecting & 0 & 0 & 2 & 0 & 3 & 0 & 2 & 0 & 0 & 2 & 0 & 1 & 0 & 4 & 2 & 1 \\
\hline Moving & Naming & 8 & 5 & 9 & 19 & 14 & 4 & 10 & 15 & 9 & 7 & 6 & 5 & 16 & 12 & 9 & 8 \\
\hline
\end{tabular}




\begin{tabular}{cc|c|c|c|c|c|c|c|c|c|c|c|c|c|c|c|c}
\hline Framing & 2 & 1 & 5 & 2 & 2 & 4 & 2 & 2 & 0 & 4 & 1 & 2 & 4 & 7 & 2 & 4 \\
\hline Moving & 9 & 28 & 19 & 31 & 7 & 18 & 11 & 14 & 12 & 27 & 13 & 19 & 16 & 16 & 18 & 20 \\
\hline Reflecting & 14 & 22 & 11 & 20 & 9 & 18 & 13 & 16 & 15 & 19 & 17 & 15 & 12 & 13 & 15 & 17 \\
\hline Naming & 9 & 9 & 12 & 11 & 6 & 6 & 12 & 8 & 5 & 4 & 7 & 12 & 5 & 2 & 8 & 4 \\
\hline \\
Reflecting Framing
\end{tabular}

Table 12 Summary of Results

Comparison
Type

The digital sketching condition resulted in significantly increased time spent focused on

TDR-Focus 1 1. the tool of design representation. Conversely, conventional sketching resulted in significantly more time spent in design focused work.

\begin{tabular}{|c|c|c|}
\hline $\begin{array}{l}\text { Implications } \\
\text { for reflective } \\
\text { practice }\end{array}$ & & $\begin{array}{l}\text { The digital sketching condition decreased non-TDR naming, moving \& reflecting } \\
\text { activities compared to conventional sketching. This reduced time spent naming important } \\
\text { considerations within the problem space, reflecting upon and exploring the } \\
\text { appropriateness of ideation attempts through design-driven moving activity. }\end{array}$ \\
\hline (Distributions) & 2. & $\begin{array}{l}\text { The frequencies of TDR-focused moving and reflecting activities were significantly } \\
\text { increased in the digital sketching condition, resulting in greater time spent exploring tool } \\
\text { functionality and reflecting upon the appropriateness of the tool or tool function, limiting. }\end{array}$ \\
\hline & 3. & Digital sketching reduced transitions between naming and framing. \\
\hline Transitions & 4. & $\begin{array}{l}\text { The use of the digital sketching increased transitions between moving and reflecting, and } \\
\text { moving to moving. }\end{array}$ \\
\hline
\end{tabular}

